\title{
A supervised machine-learning method for optimizing the automatic transmission system of wind
} turbines

\author{
Habeeb A. H. R. Aladwani ${ }^{*}$, Mohd Khairol Anuar Ariffin ${ }^{a}$ and Faizal Mustaphab
}

${ }^{a}$ Department of Mechanical and Manufacturing Engineering, Universiti Putra Malaysia, 43400 UPM, Serdang Selangor, Malaysia ${ }^{b}$ Department of Aerospace Engineering, Universiti Putra Malaysia, 43400 UPM, Serdang Selangor, Malaysia

\begin{tabular}{l}
\hline A R T I C L E I N F O \\
\hline Article history: \\
Received 21 June 2021 \\
Accepted 26 November 2021 \\
Available online \\
27 November 2021 \\
\hline Keywords: \\
Wind Turbine \\
Automatic Transmission System \\
Machine-learning \\
Energy Loss \\
Python
\end{tabular}

\section{Introduction}

In recent years, Wind Power is considered the world's fastest-growing source of renewable energy. Currently, wind energy is the second-largest source of renewable energy, and by the year 2035, wind energy will take up to $25 \%$ of total renewable energy, according to the Global Wind Energy Council (GWEC)'s 2013 wind report. Traditionally, wind turbines are located onshore, but lately, they are increasingly installed offshore due to community demands. A turbine can produce electricity up to 70-85 percent of the time, reliant on local wind speed. Wind energy is highly demanded as it is sustainable and environmentally friendly (Update, 2017). For wind energy, wind turbine applications need to be appropriately selected. This innovation has broadened to a few world areas and created a great foundation with comparative costs. There are three types of wind turbines classified based on the shaft orientation and axis of rotation: horizontal axis wind turbines, vertical axis wind turbines, and ducted wind turbines. The first type of wind turbine, the Horizontal axis wind turbine (HAWT), is a turbine with a shaft-mounted horizontally parallel to the ground. This type of wind turbine is more commonly used. The second type of wind turbine is the vertical axis wind turbine (VAWT), which its shaft is normal to the ground. This type of wind turbine is less frequently used which Savonius and Darrieus are the most common in the group. The third wind turbine can be either horizontal or vertical axis, but the turbine blades are encased in a shroud or hollow-shaped duct and known as a Ducted Wind Turbine (DWT). These wind turbines are mainly used for electricity generation.

* Corresponding author.

E-mail addresses: aladwanihabib@gmail.com (H. A. H. R. Aladwani) 
The VAWT is not as regular and has just as of late been utilized for a huge-scale power era. A few studies have shown that the purpose of the VAWT offers more favorable circumstances than the HAWT. The VAWT does not require to be orientated to the course of the wind. Other than that, it does not need to be bothered with a tower, thus decreasing capital costs. The generator is mounted at ground level to ease access (Al-Bahadly, 2009; Kanellos \& Hatziargyriou, 2008; Yeh \& Wang, 2008). In this research, Vertical Axis Wind Turbine is designed with an automatic transmission system to improve electric generation efficiency. The reliability of wind turbines is a prerequisite to ensure the healthy growth of wind energy. Even if certification bodies validate new designs and prototypes performed by manufacturers and could offer safer and more reliable wind turbines, their development and related improvements are still based on the experience with smaller turbines than those currently being erected. Therefore, the technology is still coming up against its limits. To this end, it has been recognized that there is a need to continuously monitor major wind turbine components such as gearbox, generator, and rotor blades. These components are seen to require substantial maintenance and repair efforts or even retrofits (Nivedh, 2014). Verma and Pachori (2015) stated that wind turbines could either operate at fixed speed or variable speed. For a fixed-speed wind turbine, the generator is directly connected to the electrical grid. The most common type of wind turbine is the fixed-speed wind turbine with the induction generator directly connected to the grid. This system has several drawbacks, however. The reactive power and, therefore, the grid voltage level cannot be controlled. Most of the drawbacks of a fixed wind turbine are avoided when variable-speed wind turbines are used. These turbines improve the dynamic behavior of the turbine and reduce the noise at low wind speeds. For a variable speed wind turbine, the generator is controlled by power electronic equipment. There are several reasons for using the variable-speed operation of wind turbines; among those are possibilities to reduce stresses of the mechanical structure, acoustic noise reduction and the possibility to control active and reactive power. However, in variable speed wind turbines, there are losses in power electronics and some components. It can even cause an increase in the cost of equipment because of the power electronics (Verma \& Pachori, 2015).

In this research, the experimental VAWT is a fixed-speed wind turbine. The grid voltage level cannot be controlled since the generator is directly connected to the grid by referring to Verma and Pachori (2015). These problems lead to some issues as follows:

The current wind turbine only has a one-speed gearing system which when the wind speed is inconsistent, the gearbox is not capable of varying the gear ratio, which contributes to the gears cracking and gearbox failures (Cho et al., 2015). The current gearbox systems cannot vary the gear ratio due to the one-speed gearing system, leading to less efficiency in harvesting the power from wind energy. Hence, energy loss occurred. This research aims to design an automatic control transmission system for vertical axis wind turbines using the machine learning algorithms in a way that the system can forecast the wind speed changes according to its database and then change the wind turbine gears accordingly. The scope of this research is covering the application of automatic transmission systems by using a centrifugal clutch, not Continuously Variable Transmission (CVT) or planetary gear.

The rest of the paper is organized as followings:

In the next section, an in-depth review on the literature of wind turbines will be carried out. Then, in the research methodology section (section 3), an automatic transmission system for vertical axis wind turbines will be designed first. Then, in the second phase, a supervised machine learning algorithm will be proposed to let the system learn the environment data to automatically change the gear and turbine speed in line with the wind speed. Then, in the results and discussion section (section 4), the results of simulating the wind speed on voltage and power of the designed automatic transmission system will be simulated and analysed. Then, the results from the testing of the vertical axis wind turbine with automatic transmission system will be compared to the vertical axis wind turbine without automatic transmission system. Then, recommendations for future studies will also be suggested.

\section{Literature Review}

The most renewable form of energy is sun-based energy, as it can be utilized specifically for warmth or power. Hydropower originates from falling water because the sun-based energy will cause water to vanish at low rise and rain at higher heights. The sun plays its role in warming different parts of the world's surface. Biomass energy originates from plant matter and is delivered through photosynthesis which is driven by the sun. In this manner, biomass, wind, and hydropower are simply secondary sources of sun-based energy. Non-sun-based renewable energy sources incorporate geothermal energy, which originates from the center of the earth, in some form of mixed energy from the beginning and then proceeded with the root of atomic materials.

Biomass is fuel obtained from organic matter combined with wood, crops, trim developments, and animal waste. Fossil fuel is similar to biomass but is out-of-date. Biomass is humankind's imperative source, utilized ever since the revelation of fire. Despite all that, it is $10 \%$ of the world's basic vital supply and the world's greatest single renewable essential source, as a critical part of the aggregate mass uses wood, charcoal, straw, or animal fertilizer as a cooking fuel (IEA 2012). 


\subsection{Wind Power}

Like biomass, wind control had also been used some time ago. In the best areas, introducing the day control era of twist is close cost uniformity with power sources, such as coal and nuclear. Regardless, there is a significant complexity between wind control cost on the best goals and less sensible regions. Wind power is created by the energy of moving air and varies with the robust state of wind speed. Duplicating wind speed has $2 \times 2 \times 2=8$ times more potential energy; tripling wind speed achieves $27\left(3^{3}\right)$ times more energy. More potential energy, of all things considered, means cutting down costs for a given measure of energy. The windiest conditions are therefore much better than less tempestuous regions. Generally, these districts are in shoreline fronts and offshores, along mountain edges, and in boundless open extents like the US Unprecedented Plains. Wind control potential in numerous zones is constrained and confined by the number of conditions where the energy source can be made at a reasonable cost. In any case, if the energy could be moved along the divisions, a locale like the US Amazing Plains could, on a fundamental level, supply a massive amount of energy for the US. Not simply midpoints wind control changes unfathomably by site, yet control is available at a particular moment furthermore contrasts hugely with wind speed. Significantly more essentiality is open on windy days than on calm days. This brokenness trademark is consistent with most renewable essentialness sources; however, it is particularly trying with the wind that gives how much potential imperativeness changes with wind speed. The furthest variable point of an energy plant is portrayed as the extent of honest to goodness imperativeness made to the most extraordinary essentialness era potential. Nuclear and coal control plants usually have high furthest point factors, at times outperforming $90 \%$, inferring that over a year, they can make over $90 \%$ of the energy they would get from constantly running at the most extraordinary yield for a whole year. A breaking point considered for bend control on a fair site might be $30 \%$ and much lower in poor wind districts. While wind power is occasionally criticized because of its unavoidably low cut-off figure, this is only an issue relating to cost. Like all energy sources, wind control has its specific externalities. The ones of concern are the impact of the wind turbines, which are usually more than 400' in stature; tumults related to the bent forefronts in the turbine, which can be troublesome when in close proximity to the wind turbines; and feathered animal mortality caused by accidents with the sharp turbine edges. The legitimate siting of wind workplaces may mitigate noise and the death of winged animals. However, wind power is not adaptable in siting, especially in the windiest territories. The impact is not easily directed, as wind power requires broad structures that are not adequately concealed. In any case, possibly all radiance is subjective, and we may find wind turbines to be engaging, to some degree, in perspective of the renewable energy they address. Ocean wind energy is a renewable energy resource with the potential for less negative externalities than inland. Wave power can be seen as a supporting source of contorting power, as wind produces waves. Procedures for saddling wave control consolidate floatation contraptions that rise and fall with the waves, making mechanical energy change over to control at last. While there are various established blueprints for handling wave energy, there are several huge-scale working cases. Again, the cost is the issue: while some energy is available in the waves, it is expensive to change this into accommodating energy for society.

Table 1. Comparison between VAWTs and HAWTs (Bhutta et al., 2012)

\begin{tabular}{lll}
\hline & VAWT & HAWT \\
\hline Tower sway & Small & Large \\
Yaw mechanism & No & Yes \\
Self-starting & No & Yes \\
Overall formation & Simple & Complex \\
Generator location & On ground & Not On-ground \\
Height from ground & Small & Large \\
Blade's operation space & Small & Large \\
Noise produced & Less & Relatively high \\
Wind direction & Independent & Dependent \\
Obstruction for birds & Less & High \\
Ideal efficiency & More than $70 \%$ & $50-60 \%$ \\
Wind velocity for start & Very low & Relatively high \\
\hline
\end{tabular}

Universally, wind energy has a significant potential to become renewable energy. It can supply more than two-fold of the present politically influenced nation use, which is around $15,000 \times 109 \mathrm{kWh}$ consistently. The supply can consistently finish from $20,000 \times 109-50,000 \times 109 \mathrm{kWh}$ (Hashim \& Ho, 2011; Herbert et al., 2007). It depends on a couple of parts, for instance, the typical wind speed, the exact wind speed transport, turbulence powers and the cost of wind turbine systems. Other than this, more than 50 countries and 1,500 affiliations contribute to hardware manufacturing, broadening headways, controlling time, storage, and consultancy. Such a gigantic number accelerates the updates and movements of wind energy advancement. Wind turbines can be arranged into two main types, depending on the bearing of the rotor shaft. One is the Horizontal Axis Wind Turbine (HAWT) and the second type is the Vertical Axis Wind Turbine (VAWT). HAWTs have sharp edges mounted radially from the rotor. Today, most HAWTs have sharp edges and are usually utilized for an expansive scale of network electrical power. VAWTs are not as basic and have just been recently utilized for substantial scale power. Both wind turbine types have been thoroughly tested and improved (Herbert et al., 2007). Several studies found that the use of the VAWT has more points of interest as compared to the HAWT (Bhutta et al., 2012; Dabiri, 2011; Eriksson et al., 2008; Mohamed, 2012). A comparison between the VAWT and the HAWT is presented in Table 1. The VAWT does not have to be orientated to the wind direction and it does not need a tower, reducing capital costs. The generator needs only to be mounted at ground level for easy access (Kanellos \& Hatziargyriou, 2008; Yeh \& Wang, 2008). Additionally, recent studies show that VAWTs can be 
installed much closer to each other compared to HAWTs, and because of this, the power density per square meter could be considerably higher than for the configurations used presently(Dabiri, 2011). For various reasons, there is now a resurgence of interest in VAWTs, in particular Darrieus turbines (Mohamed, 2012). Furthermore, VAWTs exhibit more advantages compared to HAWTs in terms of Malaysia's weather conditions and risks. They harmonize with the circumstances, such as low average wind velocity and risks of lightning and bird strike. The types of VAWT are further analyzed and reviewed in the next paragraph.

The investigation of VAWT design has been directed by now and is being built up. There are a few setups recorded as follows: the Darrieus rotor - egg blender formed, the Darrieus rotor - straight bladed, the Darrieus rotor - variable geometry oval direction (VGOT), the Darrieus -Masgrowe, the turned three-bladed Darrieus rotor, the Crossflex, Savonius rotor, the Combined Savonius and Darrieus rotor, the two leafed semi rotating, Sistan wind process and the Zephyr turbine. As far as the assembling procedure and manufacturing costs are concerned, the Darrieus rotor-straight cutting edge or giro mill demonstrated a dependable arrangement. The two cutting edges of the giro mill are, for the most part, named the H-rotor (Howell et al., 2010; Mertens et al., 2003). Consequently, profound thought and survey will be directed on the Darrieus rotorstraight sharp edge. As far as the execution of the VAWT, the focal shaft delivered a higher effect of the vibration. Other than this, the productivity of the VAWT is expanded by the more noteworthy length of the VAWT and the breadth of the turbine edges. Previous studies on VAWT execution concentrated on torque (Chong, Fazlizan, et al., 2012; Gavalda et al., 1990; Islam et al., 2008; Mohamed, 2012; Park et al., 2012), control (Chong, Poh, et al., 2012; Gavalda et al., 1990; Greenblatt et al., 2012; Hossain et al., 2007; Mohamed, 2012; Park et al., 2012) and rotational speed (Chong, Poh, et al., 2012). A few components impacting the VAWT execution were raised, for example, sharp edge wellbeing, liquid stream around the edges, and the outline of the wind turbine. The studies were directed towards utilizing reproduction and trial strategies and the improvements of the VAWT execution. In a plan examination, the outline arrangements may incorporate a few existing VAWT types (Gavalda et al., 1990). Another outline of the Darrieus and Savonius joined rotor is proposed and examined. The beginning of the torque demonstrates a change. An examination of the existing standard air foil shapes led to 20 plans (Mohamed, 2012). From the 20 outlines proposed, they were subjected to computational investigations, where the H-rotor Darrieus turbine (including the S-1046 type of air foil) gives off an impression of being highly appropriate to the wind energy conditions, especially in urban regions. (Greenblatt et al., 2012) proposed a plasma actuator to control the stream partitions, which expanded the power created to around $38 \%$. Stable and adjustable pitch edges were additionally examined. The outcomes demonstrated how the adjustable pitch edges could beat the beginning torque issues connected with the VAWTs. Besides this, extra adornments may also help in refining the measure of force created. The guide vane placed at the external gadget of the VAWT framework may go about as the Bernoulli rule. The diminishment in pneumatic force causes the air to stream into the passage at a higher speed than the external speed. It enhances the rotational speed and the beginning of the conduct execution (Chong, Fazlizan, et al., 2012; Chong et al., 2011; Chong et al., 2013; Chong, Poh, et al., 2012; Takao et al., 2009). The trial yield was likewise upheld and enhanced by the reproduction examination. The examination on vortex reproduction, dynamic slow down and tallness to measurement proportion demonstrated a superior clarification and comprehension of the streamlined issue in the test (Islam et al., 2007; Stein et al., 2012; Vandenberghe \& Dick, 1987). In examining the edges, the impact of a defective sharp edge on the torque and power yield were considered. It demonstrates that the torque and power could diminish as the quantity of missing sharp edges expands (Park et al., 2012). Both exploratory and reproduction techniques were led to extend the innovation of VAWTs, particularly for RE fields. The plan investigation appears to have achieved some kind of development. Scientists have also carefully considered the parameters included, for example, streamlined execution and liquid stream investigation. Be that as it may, there are still research chances to be sought after in enhancing a sharp edge plan for a few components, for example, traverse length, harmony length, producing capacity and streamlined shapes. Advanced investigation can likewise be directed to examine variables that are most powerful in a VAWT execution. In any case, the studies on auxiliary honesty should be researched further; the issues highlighted in basic trustworthiness include the structure's basic point, sharp edge vibration usage, the impact of the progression of twist stream in a brief period, the basic wellbeing observing framework, the normal recurrence of the structure and material choice.

The Horizontal Axis Wind Turbine (HAWT) is the most widely recognized and can often be seen littered over the scene in territories of similarly level ground with unsurprising year-round wind conditions. HAWT sits on an extraordinary segment and holds a great deal of cutting edge that rotates around a pivot parallel to the present course. These wind turbines have been the essential issue of wind turbine investigation for quite some time, fundamentally because they impart basic operations and elements to revolving airplanes. In HAWT, there are two essential types: the wind turbine that faces the wind and the other one that faces far from the wind. Turbines that face into the wind require a rudder or some other kind of instrument to have the capacity to self-orientate in confronting the approaching current of air. Those that face far from the wind need not bother with this per user to self-orientate; in any case, they experience the ill effects of vibration because of the bolster tower blocking part of the wind stream. While the Vertical Axis Wind Turbine (VAWT) pivot around a hub opposite the approaching stream, they can subsequently take a twist from any headings, leading them to be more flexible. VAWTs are rough, calm, omnidirectional, and they do not place as much weight on the bolster structure. They can be pressed nearer together in wind ranches, permitting more in a given space. It is not because they are smaller but also due to the moderating impact reported in real-time, compelling originators to separate them by ten times their width. The VAWT is not as proficient as the HAWT and it has low beginning torque. Not only it has dynamic soundness issues, but also it offers benefits in low wind circumstances where the conventional HAWT experiences issues when working. The fundamental advantages are acquired and enhanced 
execution at lower wind speeds and a lower r.p.m. administration at higher wind speeds bringing about a quiet turbine appropriate for private situations. Likewise, it tends to be more secure and simpler to construct, and it can be amassed near the ground and can handle turbulence much superior to the flat ones (Polinder et al., 2004).

VAWTs comprise two noteworthy types, the Darrieus rotor and Savonius rotor. The Darrieus wind turbine is a VAWT that pivots about a focal hub because of the lifts delivered by the turning surfaces, though a Savonius rotor pivots because of the drag made by its sharp edges. There is in like manner a crude sort of VAWT developing in the wind control industry, which is a blend between the Darrieus and Savonius plans. There are two major configurations of VAWTs: The Savonius and Darrieus rotor types. The earlier design is a drag-type turbine, where power is generated using momentum transfer. In contrast to the Savonius formation, the Darrieus formation is a lift-driven turbine: The power is produced from the aerodynamic torque acting on the rotor (Korobenko et al., 2014). The efficiency of Darrieus-type turbines is significantly higher than the one of the Savonius type, and because of this, the Darrieus-type turbines are chosen in this research study. However, a detailed study will still be done for both designs to understand the wind turbines' design appropriately. As energy resources in the world are continuously depleting, the importance of green energy keeps rising every day. Malaysia is a country that is blessed with not just windy beaches around the country but also airports surrounded by massive and empty land areas. It should be optimized by this gift and start considering wind energy as one of the energy sources. It can significantly boost Malaysian airports' performance and ranking in general and other related institutions such as aircraft maintenance training and renewable energy institutions in this country. However, currently, there is a lack of research in wind turbines, especially focusing on comparing HAWT and VAWT performance in this country. This study is focused on building the functional wind turbines and comparing the performance of HAWT and VAWT under certain wind speeds and behaviors, which is just one of the aspects that need to be considered. In terms of power generation under a steady wind stream, HAWT is the better one. The HAWT can produce much higher energy in steady and high wind streams. However, this is not a feasible form of analysis because the two types of wind turbines are not comparable. The huge gap in current and voltage output is due to the major weight difference between both wind turbines. The weight of the VAWT is almost double the weight of the HAWT. This factor affects the prototype's performance as more wind speed is needed to turn heavier blade sections. A better comparison can be made based on the second indoor testing: wind angle change. VAWT is efficient in this environment where the direction of the wind is changing. While the performance of HAWT is dropping as the direction of the wind is away from the direction of the blade, VAWT is capable of maintaining the output throughout the test. In a sporadic environment, HAWT faces difficulties in responding while VAWT flourishes in turbulent and sporadic wind patterns. In Malaysia, where the wind direction is unpredictable, a VAWT with improvable performance would most likely perform better than the HAWT due to its handling of turbulent and omnidirectional wind (Johari et al., 2018).

\subsubsection{Wind Energy Harvesting}

Harnessing wind energy provides a means to reduce dependencies on fossil fuel reserves. With the rapid growth of the global human population, the energy demand also increases. Therefore, many countries worldwide have adopted renewable energy technology to generate clean and inexhaustible energy to fulfill their ever-increasing electricity demands. In some places, $100 \%$ of their average yearly demand is provided by renewable energy resources. As one of the fastest-growing renewable energy resources in the world today (Gipe, 2004), the global cumulative installed wind capacity has increased significantly since 1996 . The total wind power capacity at the end of 2015 was about $433 \mathrm{GW}$, as reported in Figure 2.12 . It is projected to reach $2000 \mathrm{GW}$ by 2030 , supplying between 17 and $19 \%$ of global electricity demand. There are two major types of wind turbines; the horizontal axis wind turbine (HAWT) and the vertical axis wind turbine (VAWT). In general, the HAWTs are better at extracting wind energy than the VAWT. Therefore, most wind turbines in the commercial market today are dominated by HAWT machines. However, researchers and manufacturer's new interests in the VAWT technology have reignited major development efforts for this wind turbine (Global Wind Energy Council, 2015). In some situations, the VAWT has superior advantages over the HAWT, including its ability to extract wind energy from almost every direction, is easier to maintain, has less visual impact, produces low noise emissions, and works with improved performance in skewed wind flow conditions. The complex characteristic of urban winds involves erratic, insubstantial and inconsistent wind flow due to the many obstacles (i.e., buildings). The distinctive characteristic of urban wind requires wind turbines that suit this phenomenon well. The VAWT is deemed more suitable for the urban context than the large and more common HAWT.

\subsubsection{Automatic Transmission Systems}

Ariffin et al. (2014) stated that a remote-controlled vehicle's transmission system is as same as an automated manual transmission system in a life-sized car, in which the transmission included an automatic clutch assembly. The automatic clutch assembly aims to perform a clutch engaging operation automatically by an actuator for a clutch. The automated manual transmission is connected to an engine (Ariffin et al., 2014). In an automated manual transmission, an actuator shifts from one gear to another in response to the sleeve's axial movement. Alternatively, in this transmission, the actuator was replaced by the centrifugal clutch to shift one gear to another gear, thus regulating the engine's torque. 


\subsubsection{Automatic Gearbox}

According to Raut and Mali (2014), the automatic gearbox consists of an assembly of gears and centrifugal clutches. In their work, an automatic gearbox applied with centrifugal clutches has been designed and manufactured. Fig. 1 shows the schematic diagram of the Automatic Transmission System Gearbox with centrifugal clutches. The A \& B are respectively driving and driven shafts. Driving and driving shafts are parallel to each other. These two shafts are carrying all the rotating elements. The most important thing is that the main principle of an automatic transmission system depends on the centrifugal clutch. Three clutch drums C1, C2 \& C3 were applied to the system. On each of the centrifugal clutch hubs, the pinion gear was fitted. This means, on the hub of clutch drum D1, Pinion Gear P1 is fitted. While, on the hub of clutch drum D2, Pinion Gear P2 is fitted, and on the hub of clutch drum D3, the Pinion Gear P3 is fitted. These three drums are mounted on a driving shaft that is free to rotate on it. Centrifugal clutch spiders are fitted inside all the clutch drums. The clutch springs were also designed so that the clutch spiders fly apart when RPM reaches 500 for $\mathrm{C} 1,1000$ for $\mathrm{C} 2$, and 1600 for C3, respectively, hence engaging the particular gears once the required speed is achieved. The Pinions are in constant mesh with gears, which are mounted on freewheels that are fitted on the driven shaft. Figure 2.24 shows the actual model of automatic transmission gearbox with centrifugal clutches (Raut \& Mali, 2014).

\subsection{Machine Learning Algorithms}

Machine learning methods are widely used during the last decade, specifically by emerging industry 4.0. Machine learning algorithms can be used for two main purposes: pattern recognition and clustering. They can be divided into one of the two mentioned groups. Depending on the labels for data, machine learning groups can be considered supervised machine learning algorithms where the labels of the data are available, and unsupervised machine learning algorithms where the label of the data exists. In semi-supervised learning methods, an agent exists that can get information from the environment and use it to train the model. Fig. 1 shows some of the most frequently used supervised and unsupervised machine learning.

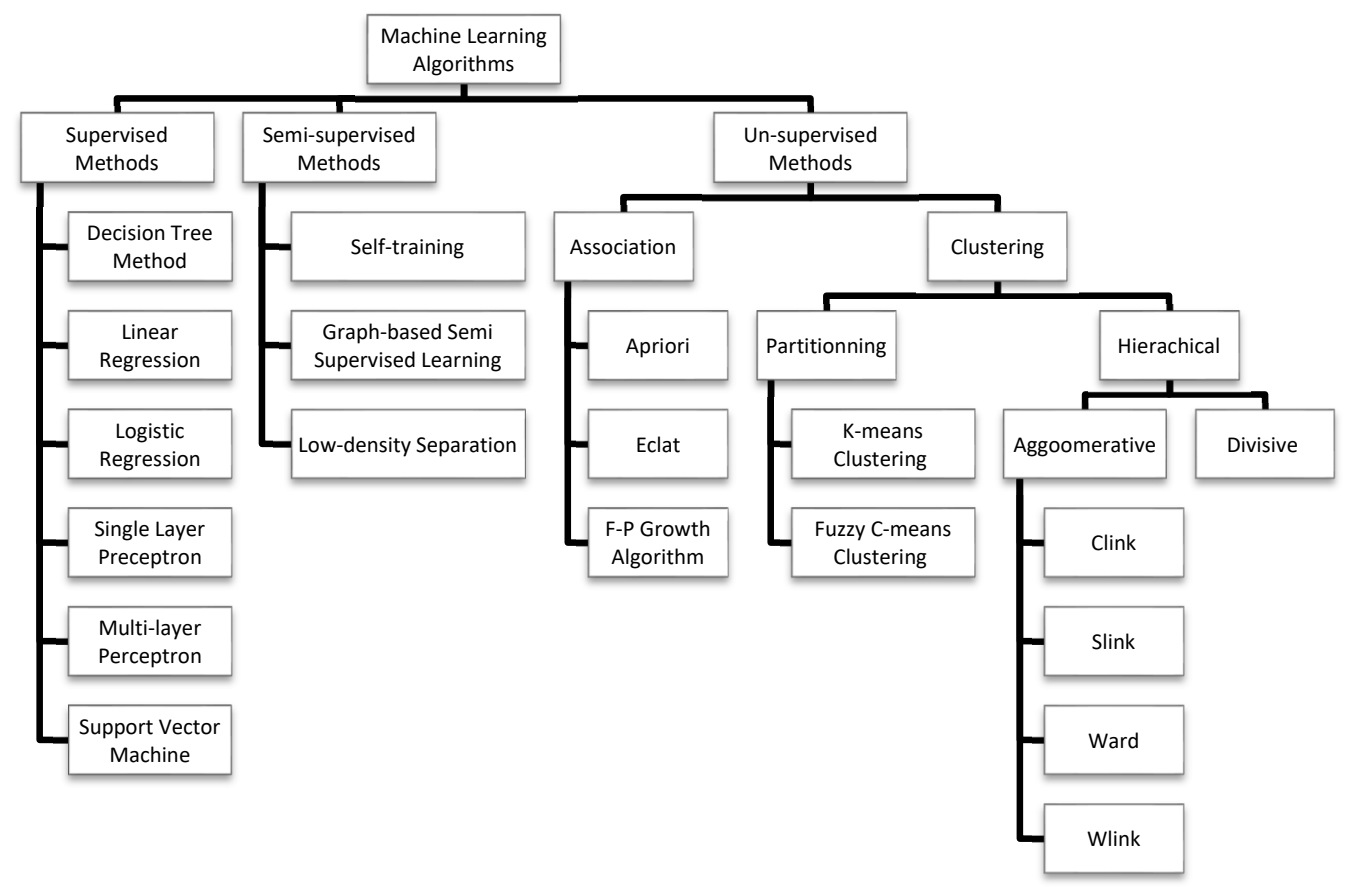

Fig. 1. The most frequently used Machine Learning algorithms

\subsection{Comparing Supervised, Unsupervised, and Reinforcement Machine Learning Algorithms}

In order to choose the best machine learning algorithm that matches the problem statement of this research, the attributes of the machine learning algorithm must be compared first. Table 2 compares the attributes of the machine learning methods: 
Table 2. Comparing Machine Learning Methods

\begin{tabular}{llll}
\hline Machine Learning Type & Label & Complexity & Training \\
\hline Supervised Learning & $\checkmark$ & Low & Using labeled data \\
Unsupervised Learning & $\mathbf{x}$ & High & Using Data Information \\
Reinforcement Learning & $\checkmark$ & Medium & Using Actions (Reward, Punishment) High \\
\hline
\end{tabular}

Today, the attention of many scholars and researchers has focused on the topic of supervised learning of systems using machine learning algorithms. Machine Learning algorithms are more in need with the growth of the market needs over the past few years, which makes them have a significant share of the countries' economy. Many researchers focused on the features of the manufacturing systems (Abecassis-Moedas, 2006; Mehrjoo \& Pasek, 2014; Nagurney \& Yu, 2012). Besides, it was shown that dynamic conditions could increase intensive entropy in manufacturing systems and influence the performance of it (Brito et al., 2015; Castillo et al., 2018; Zhou et al., 2015). In the following, a number of successful cases that used machine learning in the manufacturing systems and supply chains will be reviewed. In supply chain models, product distribution is considered a significant parameter in the success of the supply chain. Machine-learning algorithms showed excellent performance in identifying patterns in manufacturing systems and supply chains. Supervised algorithms refer to the machinelearning algorithm when the label (category) of the data for training is available. Therefore, the algorithm has a map to dedicate a series of data to a class. Several supervised machine-learning algorithms showed excellent performance in the classification of data by the learning process. Linear Regression, Logistic Regression, Support Vector Machine, Naive Bayes, Random Forest, Decision Tree, Single-Layer Perceptron, Multi-Layer Perceptron, and K-nearest Networks are among the most popular Supervised Machine-learning Algorithms. (Abbasi et al., 2019) focused on financial-based risk assessment in supply chains while the Internet of things comes into consideration. For this purpose, they used Support Vector Machine and Logistic regression methods to rate the developed risk assessment model. (Fanoodi et al., 2019) used an artificial neural network in an auto-regressive integrated moving average model for Blood products in a supply chain considering the short life span of these products. (Negrutiu et al., 2020) applied a binary logistic regression for finding the impact of features that can influence sustainable entrepreneurship in supply chains. (Mehrolia et al., 2021) used a binary Logistic Regression to classify the customers that used online food orders during the COVID-19 outbreak in India. Traditionally supervised machine learning methods are used frequently for demand forecasting. (Yue et al., 2007) used a support vector machine for forecasting demands in the retail industry where seasonal and promotional features were considered in their model. (Wu, 2010) addressed a hybrid support vector machine and particle swarm optimization for demand forecasting in the automotive industry. (Taghiyeh et al., 2020) showed that there are time series in supply chains, which correlate with geographical features. They used machinelearning algorithms for forecasting each time series in a multi-phase hierarchical decision-making method.

Carbonneau et al. (2008) presented a Multi-layer Perceptron in supply chains to minimize the bullwhip effect by forecasting demand while sufficient information about other participants' demand was not available. (Wan et al., 2016) presented the Least squares support vector machine method for electronics supply chains where interaction and added value in the supply chain were their goals. (Villegas et al., 2018) developed a new model using Support Vector Machine that uses various integrated criteria for selecting individual criteria, which showed high performance for scenarios with highly volatile demand. (Foltin et al., 2018) used KNN, decision trees, and Logistic Regression for the classification level of performance in supply chains while the capability of logistics infrastructure, the network of activities, and services operating were considered. (Mahadevan et al., 2019) reviewed machine-learning algorithms applied to the health care sector. They showed the importance of machine-learning algorithms in improving the performance of supply chains. Zhang (2019) proposed a model for predicting the exporting in the aquaculture sector. Using a multi-layer perceptron, they showed that artificial intelligence could provide an excellent method for the export prediction of aquatic products. Delgoshaei and Gomes (2019) proposed a hybrid MultiLayer Perceptron and Simulated Annealing for minimizing the cell-load variation effect in cellular manufacturing systems where their model could minimize cell underutilization using an effective preventive maintenance plan. (Liu \& Huang, 2020) addressed a hybrid ensemble support vector machine and a reducing noises method for the risk assessment process in the supply chain. (Daneshmand-Mehr et al., 2020) used Multi-Layer Perceptron to minimize the bullwhip effect in the supply chain, which causes significant shortcomings such as delayed orders or surplus inventories. Shi et al. (2021) applied a hybrid Long Short-Term Memory (LSTM) and Multi-Layer Perceptron (MLP) for predicting the geothermal productivity that could learn the non-linear relationship between the geothermal productivity and constraint conditions. Asrol et al. (2021) addressed a support vector machine algorithm for evaluating the sustainability of the manufacturing systems.

Supervised machine learning problems are successfully used for supplier selection problem in supply chains. Guo et al. (2009) addressed a hybrid potential Support Vector Machine and Decision Tree Method for supplier selection problem, which showed better performance and less calculation time than standard support vector machines. Fallahpour et al. (2018) proposed a hybrid Data Envelopment Analysis and Support Vector Machine for supplier selection where the suppliers' predicted efficiency was considered the dataset label. Delgoshaei and Gomes (2016) used multi-layer perceptron for scheduling manufacturing systems. Ishak and Wijaya (2019) used Multi-layer Perceptron for supplier selection problems in the automotive supply chains. Cheng et al. (2020) used the support vector regression method for the supplier evaluation problem in supply chains where a multiple criteria decision-making method is employed to determine suppliers' labels. Oh et al. (2019) proposed SVC for quality monitoring and control in automotive manufacturing industries, explicitly incorporating inspectionrelated expenses and error types. Jiang et al. (2019) used logistic regression for predictions of aflatoxin in grain at a postharvest stage, providing a base for guaranteeing the safety of stored grain by providing early warning on contaminant problems 
emerging while storing the product. Goettsch et al. (2020) used a multi-layer perceptron algorithm in a mathematical modeling problem where the aim was to decrease gas emissions by optimizing the biomass supply chain. There are novel research studies for using supervised machine learning methods in production planning. (Delgoshaei et al., 2016) proposed a multilayer perceptron to find the best in-house manufacturing and outsourcing quota in cellular manufacturing systems where bottleneck machines existed. Silva et al. (2017) used an ANN method to forecast the potential orders and allocate the order to the proper chain member. Golkhoo and Moselhi (2019) used a hybrid Genetic Algorithm and Multi-Layer Perceptron for material control problems in the construction sector. They showed the superiority of the proposed method compared to the classic Genetic Algorithm. Rezanoori et al. (2019) proposed an artificial neural network method for safety of passengers in an active suspension system. Kozłowski et al. (2020) addressed a Logistic Regression method for solving a model that could estimate the parameters of probabilities of transitions between operational states. From the above review, the application of automatic transmission systems in vertical axis wind turbines further investigation. There is still a lack of findings on the gearing system design and clutch design. Investigation of the gearing system and the number of the gears and clutch can be further enhanced, especially for vertical axis wind turbine applications. The application of clutch in automatic transmission systems for VAWT also needs further investigation.

This research focuses on two issues identified in the literature review. The first issue concerns using an automatic transmission system for vertical axis wind turbines to improve the efficiency of energy captured. The second issue concerns the bonding MFC design of gears and clutch for the automatic transmission system. The effect of the transmission system in VAWT is investigated. The next section will present the research methodology and the experiments employed to achieve the research objectives.

\section{Research Methodology}

The process began with a background study and reviewing the latest literature or articles related to automatic transmission systems and wind turbines. The idea of this project is to apply an automatic transmission system to wind turbines, which in this project, the vertical axis wind turbine is used. Hence, the VAWT is designed and fabricated by using the combination of Darrieus and Savonius types. As understood, the automatic gearbox with centrifugal clutches is related to an automatic transmission. More particularly, the invention describes a transmission for producing a variable speed drive. Then, the gears and clutch will be designed using gear and clutch formulas by Solidworks software and fabricated using a 3D printer. This automatic transmission system must be installed to the vertical axis wind turbine, so the wind turbine can automatically vary its gear ratio from the driving shaft to the driven shaft. The VAWT with an automatic transmission system will be tested to analyze the performance. The wind turbine needs to spin at a suitable gear ratio depending on its incoming wind speed to harvest the optimum energy. Fig. 2 showed the flow chart of the overall research.

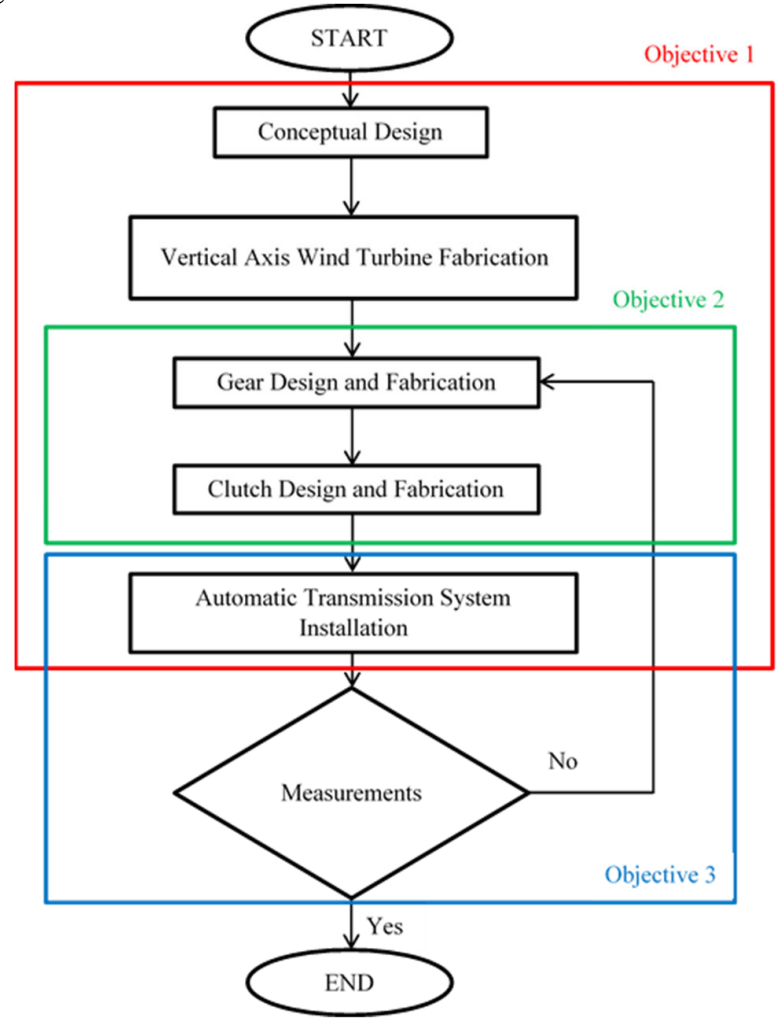

Fig. 2. Automatic Transmission System flow chart 


\subsection{Design of Vertical Axis Wind Turbine}

The Savonius is one of the simplest self-starting vertical-axis turbines. Aerodynamically, these turbines are drag-type VAWT, so they cannot rotate faster than the wind speed. It means that the tip speed ratio is smaller, making this type of turbine unsuitable for electricity generation. The Savonius turbine consists of two or three scoop rotors that look like an "S" configuration in a cross-section. The curved "S" shape allows the scoops to experience less drag force when going against the wind compared to when the scoops are moving along with the stream of the current of air. This discrepancy in drag causes the Savonius turbine to spin. The blades' cavity shape also allows the wind pressure to rotate the turbine with low speed and produces a high bending moment along the barb of the turbine because of the large area of the curved components. The efficiency of the turbines is very low compared to other types, which are approximately $15 \%$. It can then be used for other purposes, such as pumping water or grinding grain. Most of the swept area of the Savonius rotor is near the ground, creating an overall energy extraction that is less effective due to lower wind speed at lower heights. The advantages of this type of VAWTs are its simplicity, reliability, and low noise production. It can operate well at low wind speed because the torque is very high, especially in these conditions. Since the torque is not invariant, improvements such as a helical shape are used. The most ubiquitous application for the Savonius VAWT is the ventilator commonly used or discovered along the roof of vans and buses.

The Darrieus wind turbines consist of several curved airfoil blades mounted in the vertical rotating shaft or framework. The curve of the blades allows the vanes to be studied only in tension at high rotational speeds. The original design of Darrieus arranges the airfoils in symmetrical ways so that the turbine has zero rigging angles. This arrangement is equally effective no matter which direction the wind blows.

Furthermore, when the Darrieus rotor is spinning, the control surfaces move onwards through the air circularly. Proportional to the blade, the oncoming airflow is vertically added to the wind so that the resultant airflow creates a varying slight positive angle of attack to the vane. As the airfoils move around, the angle of attack changes to the opposite sign. Only the generated force is still in an indirect position to the direction of rotation because the wings are symmetrical and the ringing angle is zero. As the angle of attack changes, each blade generates its maximum torque at two spots along with its wheel, where it contributes to a sinusoidal power cycle that creates resonant modes and subsequently induces a disruption to the vane. Giromill and Cycloturbine are examples of modifications of the Darrieus turbine design. The modifications allow the generated torque to remain almost constant over a pretty wide angle, allowing it to have the advantage of self-starting. It can be done by flipping the "downwind moving" blade flat to the air current to get dragged down the turbine at low velocity. The disadvantage of these figures is that the blade pitching mechanism is difficult and laborious as it needs the wind-direction sensors to pitch the blade properly.

The vane-type wind turbine is a new type of wind turbine with a vertical area of bars in figures. It has vertical areas of three or four vanes that make a pit shape with shut vanes of the physical body to expand the drag coefficient estimate and cuts down the negative torque on the opposite side. The wind turbines have upwind rotors and utilize a tail vane for preparation into the wind. The tail vane confines the development of the rotor far from the wind. This pivoting vane framework transforms the turbine into a twist at low wind speeds and then out of the twist at higher rates. Be that as it may, at high wind speeds, it will continue delivering power. Preventing harm to the wind turbine, the sharp edges are intended to have the capacity to dislodge into a place in which they are completely or incompletely adjusted in a steady progression. This character of the wind turbine can be implicitly stated as follows: first, by tossing four edges with points, $90^{\circ}$ somewhere around one and on a level plane developed bars with vanes that can curve on $90^{\circ}$. The second case can be worked by holding only three edges with points of $120^{\circ}$ between one. Outline components should be efficiently shaped to diminish the drag constraint of the twist activity for the non-working components of a turbine. The casings are associated with the pole and the pole is associated with the electrical generator. They are secured on the bars that turn up on the sides of the suspension. Vane bars can be outlined vertically, yet in such cases, casings will have a vane flipping impact that may obstruct or reduce the dependability of a turbine. The edge's vertical parts are like a Darrieus type, where it can expand the yield of the wind turbine. During a wind condition, the vanes on the odd side of the edges are shut and will bear the twisted quality in a wide plate. The sharp edges on the correct position of the edge are uncovered and wind will fall through the open tissue. The left side vanes are visually associated with right side vanes so that vanes can be twofold driven; this development empowers the twist energy to overlap with the left side vanes and at the same time opens the right side vanes. The torque delivered by the wind constraint outlines with the yield shaft, which exchanges the torque by equipping to the electrical generator.

The wind turbine was designed by combining the Savonius and Darrieus types of wind turbines. By having a new design, the disadvantages of the two types of wind turbines can be covered by each other, hence improving the efficiency and starting torque of the wind turbine.

The Savonius type is one of the simplest self-starting vertical-axis turbines. Aerodynamically, these turbines are drag-type VAWT, which means they cannot rotate faster than the wind speed. It also means that the tip speed ratio is smaller, making it unsuitable for electricity generation. Apart from these, the Savonius turbine consists of two or three scoop rotors shaped like an "S" configuration in cross-section. Due to this curvaceous " $\mathrm{S}$ " shape, the scoops experience less drag force when going 
against the wind compared to when the scoops are moving with the current of the air. Due to this differential drag, the Savonius turbine spins. The blades are also cavity-shaped, allowing the wind pressure to rotate the turbine with low speed and producing a high bending moment along the turbine's barb due to a large area of the curved components. The turbines have low efficiency compared to the other types, which are approximately $15 \%$. Most of the swept area of the Savonius rotor is near the ground, creating a less effective overall energy extraction due to lower wind speed at lower heights. Some of the advantages of this type of VAWT are its simplicity, reliability, and low noise production. It can operate well at low wind speed as the torque is very high, especially in these conditions. Since the torque is not invariant, some improvements such as a helical shape are used. The blade of the savonius design was made by using lightweight plastic, which is Polyvinyl chloride.

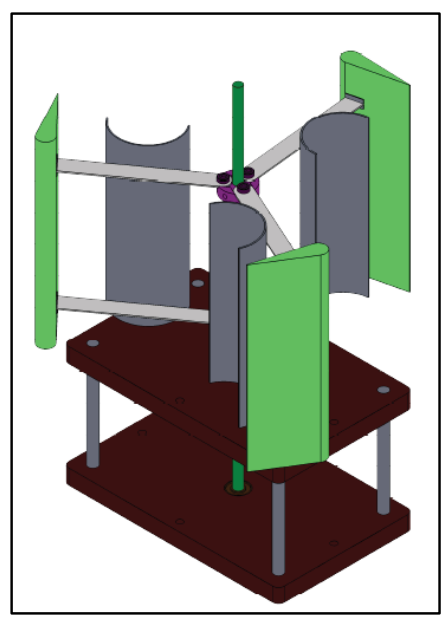

Fig. 3. Design of wind turbine

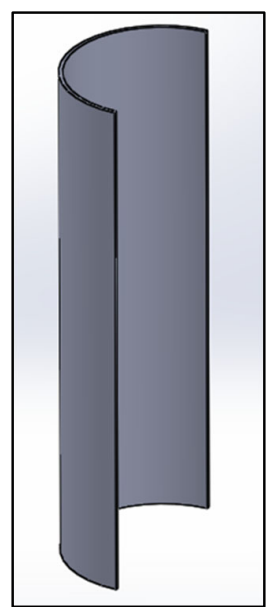

Fig. 4. Savonius blade design

The Darrieus blade is a straight air-foil blade that is mounted in a vertical rotating shaft. The aerofoils are arranged symmetrically and have zero rigging angle, which allows the turbine to spin no matter the direction of the wind. Relative to the blade, this oncoming airflow is added to the wind so that the resultant airflow creates a varying slight positive angle of attack to the blade. It generates a net force pointing forward. This force can be projected inwards past the turbine axis at a certain distance, giving a positive torque to the shaft, thus helping it rotate in the direction it is already traveling in. As the aerofoil moves around the back of the turbine, the angle of attack changes to the opposite sign, but the generated force is still obliquely in the direction of rotation because the blades are symmetrical and the rigging angle is zero. The turbine spins at a rate unrelated to the wind speed and usually many times faster. Thus, the kinetic energy from the rotation can be extracted from the torque and converted into power.

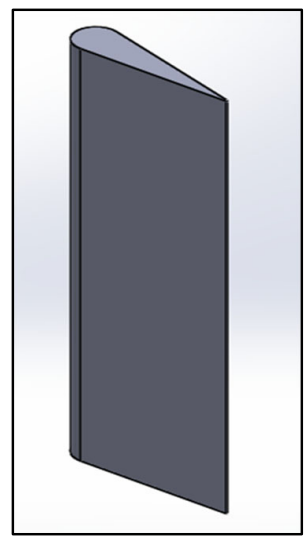

Fig. 5. Darrieus blade design

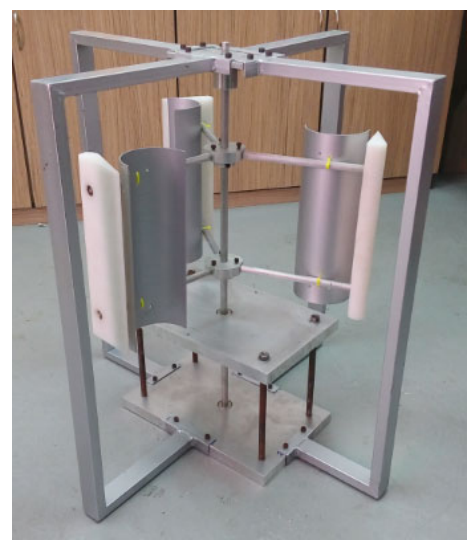

Fig. 6. Savonius-Darrieus wind turbine

\subsection{Gearing System Design}

Gearing System Design involved designing the gears, specifically the design of several teeth for the gears. The number of teeth for each gear is one of the important elements needed to be designed as it deals with gear ratio. The number of teeth for each gear can be decided by referring to the result from Table 3. At this initial stage of designing gears, some formulas and calculations were involved. The formula to calculate gear ratio is: 
Gear ratio $=\frac{\text { Maximum RPM of turbine }}{\text { RPMof turbine }}$

Each increment of incoming wind speed was set to be the desired top speed for its assigned gear; first gear, second gear, third gear and fourth gear. By referring to equation 1, the calculated gear ratios are in Table 3 as follow:

Table 3. Gear ratios and their assigned gear

\begin{tabular}{llll}
\hline Incoming Wind speed, $\mathrm{m} / \mathrm{s}$ & Revolutions per Minute $(\mathrm{RPM})$ & Gear ratio & Assigned gear \\
\hline 5 & 22.1 & 4.42 & 1 \\
10 & 47.3 & 2.07 & 2 \\
15 & 72.5 & 1.35 & 3 \\
20 & 97.7 & 1 & 4 \\
\hline
\end{tabular}

From the gear ratios calculated in Table 3, the number of teeth for each gear can be estimated by using the formula:

$$
\text { Gear ratio }=\frac{\text { Number of teeth at driving gear }}{\text { Number of teeth at driver gear }}
$$

At this stage of estimating the number of teeth, it does not have to be rigid. In order to do so, the ratio between the number of teeth at driven gear and that in driving gear needs to be "try and error" as long as they come out with the required gear ratio as calculated in Table 3. However, the limitation to consider is that the higher the number of teeth will result in a large gear size. So, in this case, the size of the gear was considered, so the gears can be fitted at the bottom part of the Vertical Axis Wind Turbine, as shown by Fig. 7.

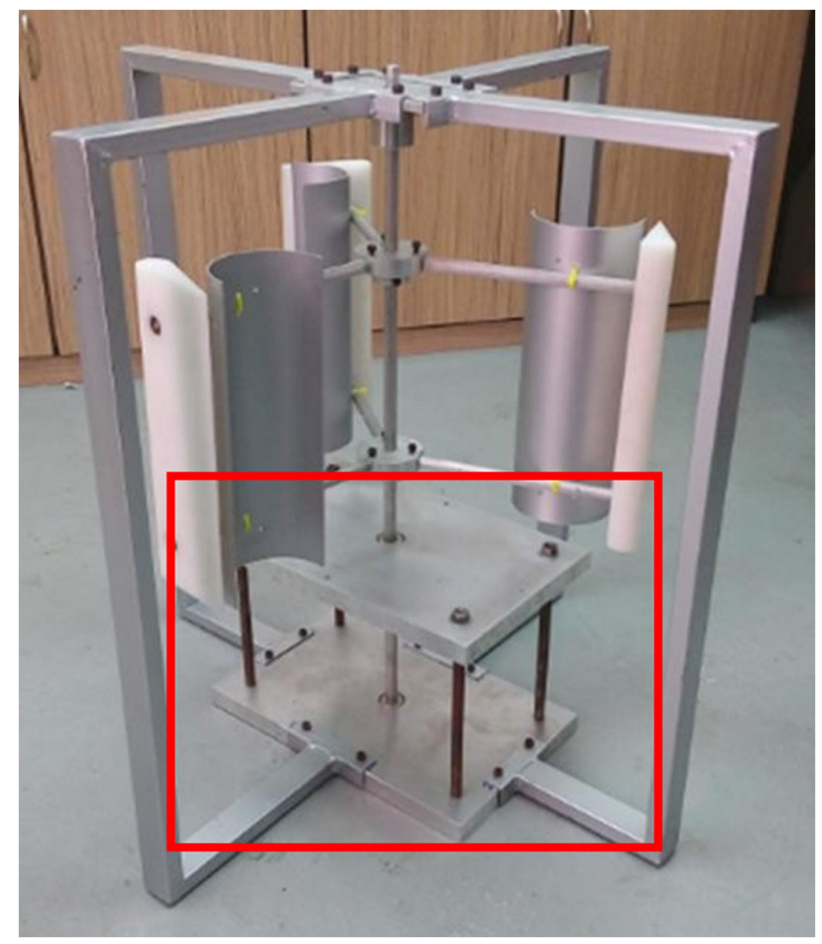

Fig. 7. Gears installation area at experimental VAWT

The calculated number of teeth at each gear is represented in Table 4:

Table 4. Number of teeth for each gear

\begin{tabular}{lllll}
\hline Assigned gear & Gear ratio & Gear action & Number of teeth & Outside diameter $(\mathrm{cm})$ \\
\hline 1 & 4.42 & Driving & 14 & 3.2 \\
& & Driven & 62 & 12.8 \\
\hline 2 & 2.07 & Driving & 15 & 5.1 \\
& & Driven & 31 & 11.56 \\
\hline
\end{tabular}


Once the number of teeth is determined, the Computer-Aided Drawing (CAD) of the gears can be designed using SOLIDWORKS. Fortunately, with the "Toolbox" function in SOLIDWORKS, the gears could be designed efficiently. The gears can be designed carefully. With the "toolbox" function in SOLIDWORKS, the user can only enter the required properties and parameters. To design the gears in SOLIDWORKS, the "Assembly" mode was selected. Then, the "Toolbox" function can be selected from the "Design Library" on the right side of the screen. Then, the spur gears were selected from the "Toolbox" function. Finally, the software required the properties and parameters such as Module, Number of Teeth, Pressure Angle, Face Width, and the gears designing was done as seen in Fig. 8.

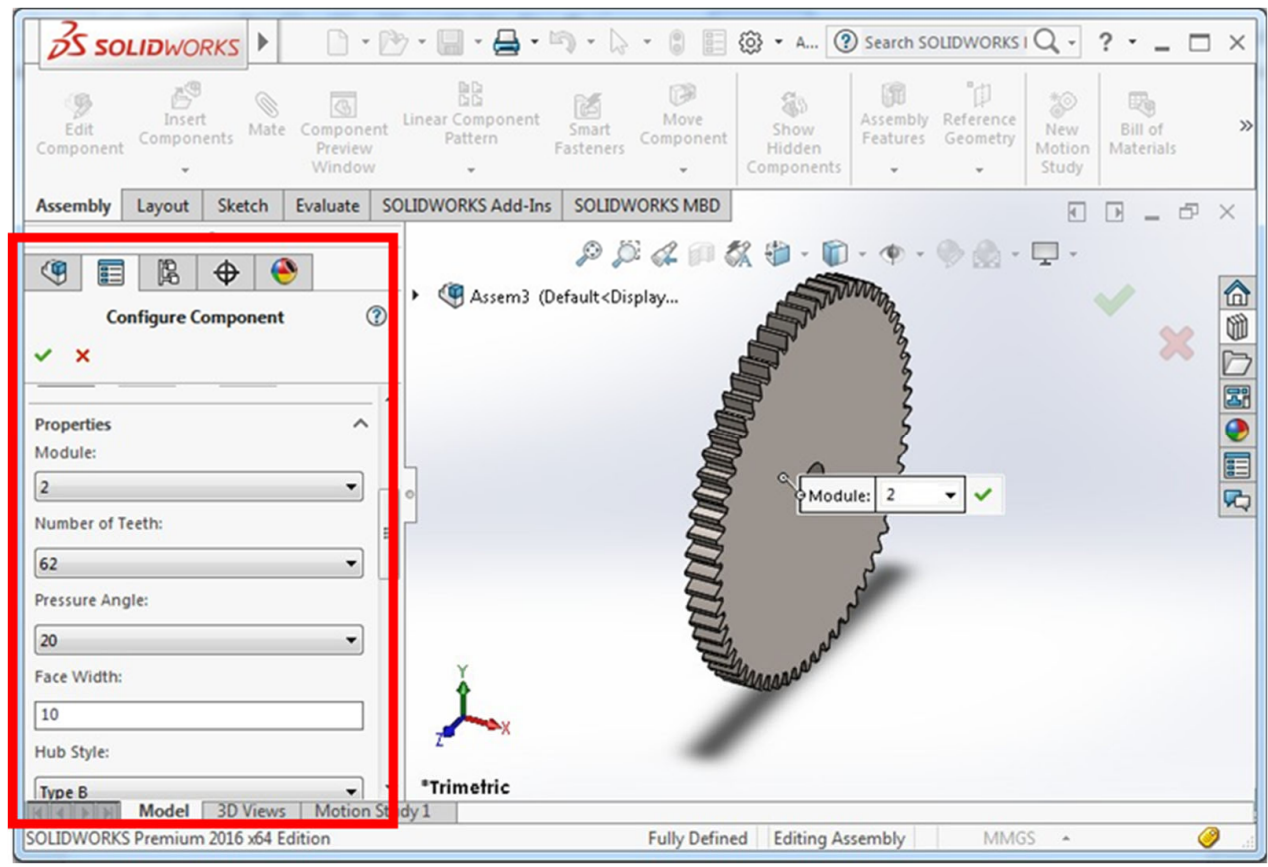

Fig. 8. Designing gears using the "toolbox" function

The properties and parameters for gear 1 are tabulated in Table 5 as follows:

Table 5. Properties and parameters for gear 1

\begin{tabular}{lll}
\hline & Driving & Driven \\
\hline Module & 2 & 2 \\
Number of teeth & 14 & 62 \\
Pressure angle & 20 & 20 \\
Face width & 10 & 10 \\
Hub Style & Type B & Type B \\
Hub Diameter & 22 & 22 \\
Overall length & 20 & 20 \\
Nominal shaft diameter & 14 & 14 \\
Keyway & None & None \\
Outside diameter $(\mathrm{cm})$ & 3.2 & 12.8 \\
\hline
\end{tabular}

For the gear 1 driving, the drawings are illustrated in Fig. 9. For the gear 1 driven, the drawings are visualized in Fig. 10 as follows: 


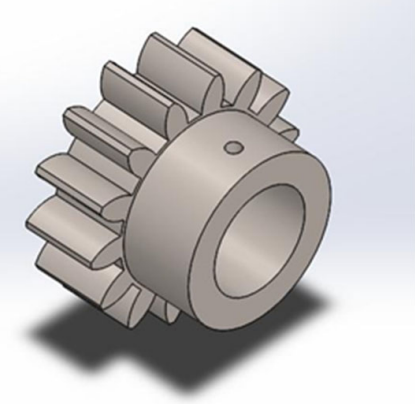

Fig. 9. Isometric view of gear 1 driving

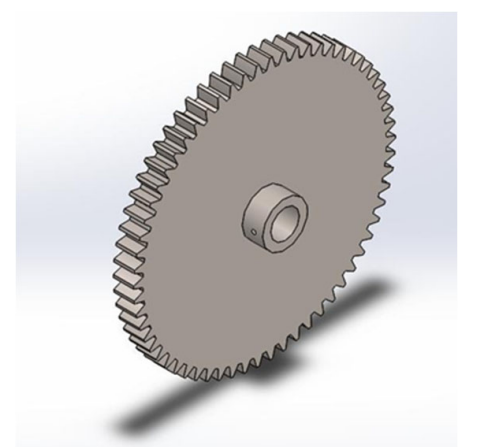

Fig. 10. Isometric view of gear 1 driven

The properties and parameters for gear 2 are tabulated in Table 6 as follows:

Table 6. Properties and parameters for gear 2

\begin{tabular}{|c|c|c|}
\hline & Driving & Driven \\
\hline Module & 2 & 2 \\
\hline Number of teeth & 15 & 31 \\
\hline Pressure angle & 20 & 20 \\
\hline Face width & 10 & 10 \\
\hline Hub Style & Type B & Type B \\
\hline Hub Diameter & 22 & 22 \\
\hline Overall length & 20 & 20 \\
\hline Nominal shaft diameter & 14 & 14 \\
\hline Keyway & None & None \\
\hline Outside diameter $(\mathrm{cm})$ & 5.1 & 11.56 \\
\hline
\end{tabular}

For the gear 2 driving and driven, the drawings are illustrated in Fig. 11 and Fig. 12 as follows:

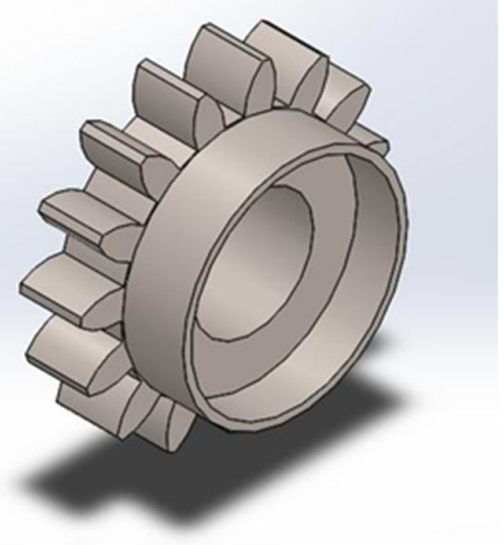

Fig. 11. Isometric view of gear 2 driving

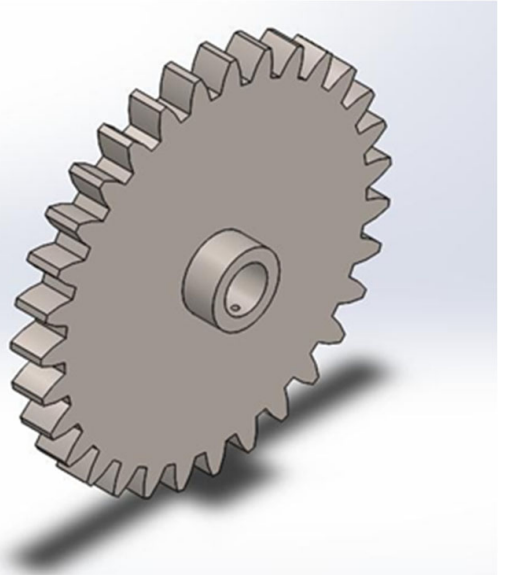

Fig. 12. Isometric view of gear 2 driven

The finalized Computer Aided Drawings (CAD) were fabricated using an Odyssey 3D printer. Before the printing process started, a few steps needed to be done. First, all the CAD files were converted to STL format. This step was to make sure that the files can be imported into Cura. Cura is the software to demonstrate how the printing process is done, as seen in Fig. 1. The STL files were imported into Cura to set the printing quality, adjust the printing speed, change the orientation of the printing object and control the fill density. Second, the CAD was then saved as Gcode file format. This step was taken so the CAD file can be imported into Pronterface. Pronterface is a printer interface for Odyssey 3D printer, as shown in Fig. 12. Pronterface was used to control the 3D printer, controlling its nozzle and platform. The Gcode file was imported into Pronterface, and the printing process started.

After modeling the gears, they need to be virtually assembled in SOLIDWORKS, as shown in Fig. 13. This step was to make sure all the gears are in the correct size to be fitted together. Other than that, by assembling, it can be seen that all the gears can be aligned correctly. Furthermore, this step was taken to visualize how this gearing system looks. 


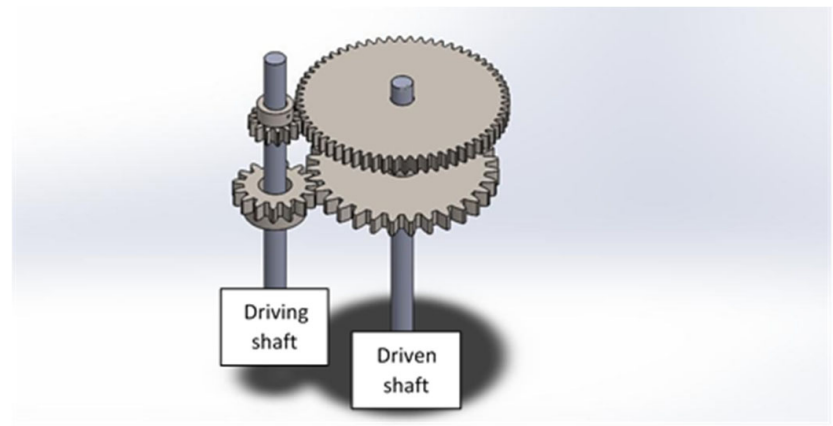

Fig. 13. Gearing system assembly

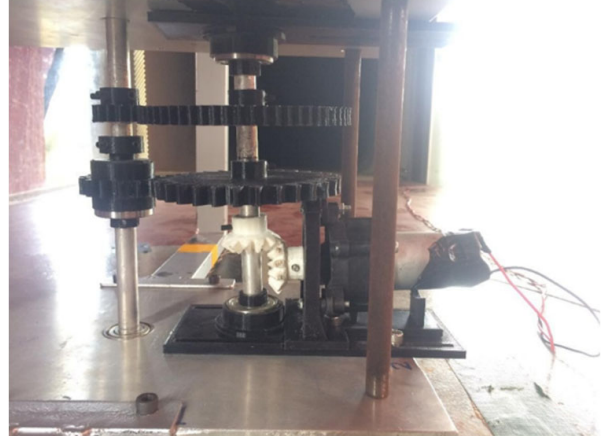

Fig. 14. Assembly of VAWT with an automatic transmission system

\subsection{A Supervised Machine Learning algorithm for Optimizing the Designed Turbine}

Random forests algorithm (R.F.), which was first proposed by (Ho, 1995), is classified among the most powerful classifying algorithms that enjoy using decision trees at the training stage for classification, regression, and various defined tasks. One dominant feature of the Random Forest Algorithm that stands out for this research is its high ability to select a class by most trees during the classification process. In other words, this method has a high capability to classify different groups with high features and is a very efficient method. The Random Forest Algorithm has been widely used in various engineering, medical and management problems. The basis of this method is in the classification of different regions by crosssupport vectors. When identifying intersecting lines, try to select the line that has the most confidence margin. The machine backup vector method uses various functions to do this. The various kernel functions used in the machine backup vector method include exponential, polynomial, and sigmoid cores.

\subsubsection{Libraries of Python}

For this purpose, the following Libraries will be used:

- $\quad$ Numpy: for support for large-scale data and multi-dimensional arrays and matrices

- $\quad$ Pandas: for using data sets and data frame

- Scipy: for modules for optimization, linear algebra, integration, interpolation, special functions

- Matplotlib: for drawing graphical views of the outcomes of the model

- $\quad$ Sklearn: for importing machine-learning algorithms tools

- Mlxtend.plotting: for drawing plot, the clusters and figures

\subsubsection{Jupyter as the Platform of Python}

Jupyter is one of the most powerful platforms for coding algorithms with Python. It is free and open-source and has been widely used throughout the world during the past years. One dominant feature of Jupyter is testing the outcome of each script line exactly below it during the coding process. It helps algorithm developers to examine each line before going further.

\subsubsection{Block Diagram of the proposed supportive vector machine algorithm}

Fig. 15 shows the process of developing the supervised machine-learning algorithm:

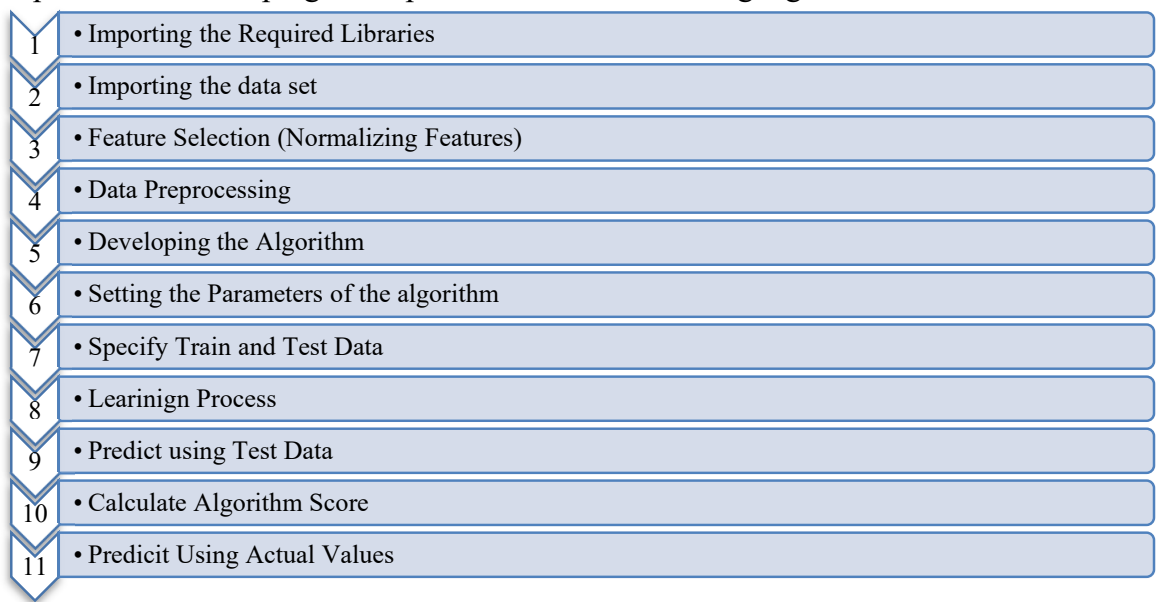

Fig. 16. The Block Diagram of the Proposed Algorithm 


\section{Results and Discussion}

This section will describe and discuss the results obtained from testing a vertical axis wind turbine without an automatic transmission system to compare with a vertical axis wind turbine with an automatic transmission system. The vertical axis wind turbine testing was conducted in a wind tunnel, and the incoming wind speed is allowed and controlled. The incoming wind speed is restricted from $0 \mathrm{~m} / \mathrm{s}$ to $20 \mathrm{~m} / \mathrm{s}$ only. The raw data was obtained from the voltage produced by the DC generator motor, where NI 9234 and NI LABVIEW were used to read the data from the generator. This section discussed output voltage and output power that the VAWT without an automatic transmission system and with an automatic transmission system could produce. The voltage data was obtained directly from the generator, while the power data was calculated using its relationship with the voltage. The calculations and formulas used were shown in this section. Then, the voltage and power produced were plotted in the form of the graph, and the plotted trend of the graphs was discussed briefly in this section. The efficiency of the energy harvesting of the vertical axis wind turbine was measured by comparing the produced voltage and power of the vertical axis wind turbine with an automatic transmission system and the vertical axis wind turbine without an automatic transmission system. The results of the comparison were discussed briefly.

In this section, a new model will be developed and solved by Random Forest Algorithm for predicting the best medicine distribution pattern among the different cities in the U.S. using Python. Python is among the most powerful applications that provide a comprehensive and robust basis for machine-learning algorithms.

The outcomes will be compared with six more supervised machine-learning algorithms in terms of accuracy score, mean of absolute error (MAE), and mean of squared errors (MSE).

\subsection{Coding the K-nearest Neighbors Algorithm}

\subsubsection{Data in Use}

This section uses a CSV file containing 30 data of testing wind turbine with automated transferring system is simulated. The dataset will be imported into Python (Table 7).

Table 7. Dataset in use

\begin{tabular}{|c|c|c|c|c|c|c|c|c|}
\hline Case & Wind Speed & RPM & Gear ratio & Time & Voltage(V) & Power(W) & VAWT Spins & $\begin{array}{l}\text { Assigned } \\
\text { gear }\end{array}$ \\
\hline 1 & 8 & 22.1 & 4.42 & 0.2 & 0 & 0.007 & 1 & 1 \\
\hline 2 & 10 & 47.3 & 2.07 & 0.4 & 0.3 & 0 & 0 & 2 \\
\hline 3 & 11 & 72.5 & 1.35 & 0.6 & 2 & 0.6 & 1 & 2 \\
\hline 4 & 13 & 97.7 & 1 & 0.8 & 5 & 2 & 1 & 2 \\
\hline 5 & 8 & 22.1 & 1 & 0.4 & 0 & 0.003 & 1 & 1 \\
\hline 6 & 10 & 47.3 & 2.07 & 1.2 & 0.4 & 0.01 & 1 & 2 \\
\hline 7 & 11 & 72.5 & 1 & 1 & 3 & 0.7 & 1 & 2 \\
\hline 8 & 10 & 47.3 & 2.07 & 0.2 & 0.2 & 0.005 & 1 & 2 \\
\hline 9 & 8 & 22.1 & 1 & 0.2 & 0 & 0.007 & 1 & 1 \\
\hline 10 & 10 & 47.3 & 2.07 & 1 & 0.3 & 0.01 & 1 & 2 \\
\hline 11 & 11 & 47.3 & 1 & 0.2 & 1 & 0.3 & 1 & 2 \\
\hline 12 & 13 & 97.7 & 1 & 1.2 & 5 & 2 & 1 & 2 \\
\hline 13 & 8 & 22.1 & 1 & 0.6 & 0 & 0.004 & 1 & 1 \\
\hline 14 & 10 & 47.3 & 2.07 & 0.8 & 0.4 & 0.01 & 1 & 2 \\
\hline 15 & 11 & 72.5 & 1 & 0.8 & 2 & 0.6 & 1 & 2 \\
\hline 16 & 13 & 97.7 & 1 & 0.6 & 4 & 2 & 1 & 2 \\
\hline 17 & 8 & 22.1 & 1 & 1 & 0.05 & 0.001 & 1 & 1 \\
\hline 18 & 10 & 47.3 & 2.07 & 0.6 & 0.3 & 0.035 & 1 & 2 \\
\hline 19 & 11 & 72.5 & 1 & 1 & 3 & 0.7 & 1 & 2 \\
\hline 20 & 10 & 47.3 & 2.07 & 0.2 & 0.1 & 0.005 & 1 & 2 \\
\hline 21 & 10 & 47.3 & 2.07 & 0.6 & 0.2 & 0.035 & 1 & 2 \\
\hline 22 & 10 & 47.3 & 2.07 & 0.4 & 0.2 & 0.01 & 1 & 2 \\
\hline 23 & 11 & 72.5 & 1 & 0.8 & 2 & 0.65 & 1 & 2 \\
\hline 24 & 13 & 97.7 & 1 & 0.2 & 4 & 1.5 & 1 & 2 \\
\hline 25 & 8 & 22.1 & 1 & 0.8 & 0.05 & 0.001 & 1 & 1 \\
\hline 26 & 10 & 47.3 & 2.07 & 0.8 & 0.3 & 0.02 & 1 & 2 \\
\hline 27 & 11 & 72.5 & 1 & 0.6 & 2 & 0.3 & 1 & 2 \\
\hline 28 & 13 & 97.7 & 1 & 0.8 & 5 & 2 & 1 & 2 \\
\hline 29 & 13 & 97.7 & 1 & 0.4 & 4 & 1.5 & 1 & 2 \\
\hline 30 & 13 & 97.7 & 1 & 1 & 5 & 2 & 1 & 2 \\
\hline
\end{tabular}

\subsubsection{Descriptive Analysis}

To continue and before any further processing, the data must be described statistically. For this purpose, descriptive analysis is done using the Pandas library, as shown in Table 17. 
Table 8 Descriptive Analysis of the Dataset

\begin{tabular}{lllllllll}
\hline & Case & $\begin{array}{l}\text { Wind } \\
\text { Speed }\end{array}$ & RPM & Gear ratio & Time & Voltage (V) & Power (W) & $\begin{array}{l}\text { VAWT } \\
\text { Spins }\end{array}$ \\
\hline count & 30 & 30 & 30 & 30 & 30 & 30 & 30 \\
gear
\end{tabular}

As shown by Table 8, all features are answered fully by the responders. Therefore, there is no need for a data pre-processing step.

\subsubsection{The Significant Features}

In this section and before choosing features to be considered in learning process, the important features must be determined. For this purpose, we used Shapiro method for ranking the features. The outcomes showed that all features are important and must be considered in the learning process (Fig. 16).

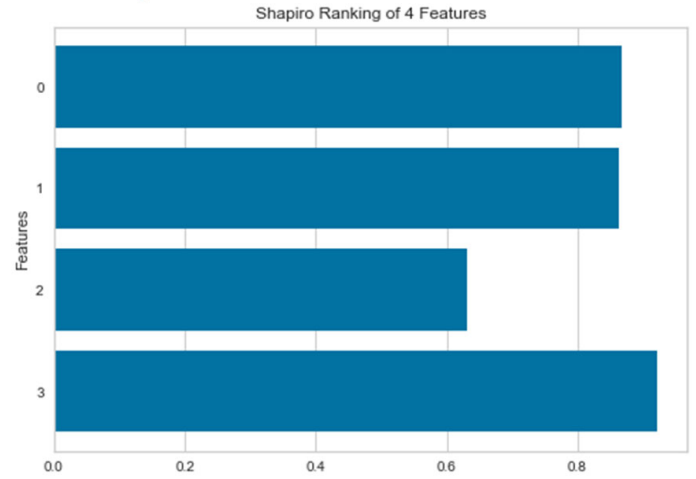

Fig. 16. Results of Sharipo Method for the Supervised Machine Learning Method

\subsubsection{Choosing Features and Labels}

In supervised machine learning algorithms before applying the fitting method, the features and label must be specified. For this purpose, the Wind Speed, RPM, Gear ratio and Time are considered as the features and voltage is considered as the label. Table 9 shows the transformed data typed into the Python.

Table 9. Data Before and After Using Standard Scaler Method

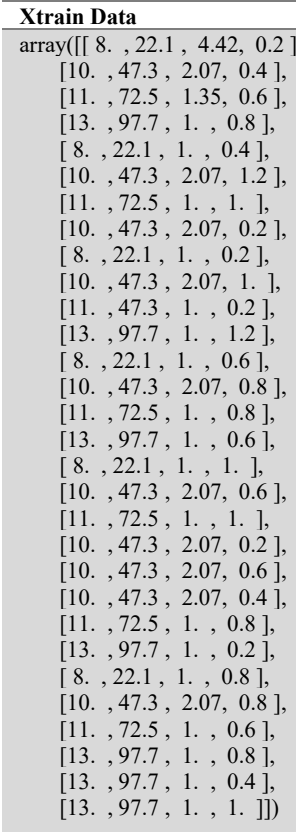

array $([$

$0 ., 0.3,2 ., 5$. , 0. , 0.4, 3. , 0.2, 0. , $0.3,1$.

5. $0 ., 0.4,2 ., 4 ., 0.05,0.3,3 ., 0.1,0.2,0.2$,

2., 4. , $0.05,0.3,2 ., 5 ., 4 ., 5]$. 
Choosing the best amount of train and test share split is vital for boosting the accuracy of the machine-learning algorithm. It can also show a valuable hint for recognizing the over-fitting or under-fitting. For this purpose, using a 'For' loop, the proposed algorithm will be run ten times. In each iteration, the algorithm chooses a specific test and train share. Fig. 17 indicates that the algorithm's performance will be maximized when the split rate is 0.2 , which provides lower possibilities of emerging under-fitting and over-fitting.

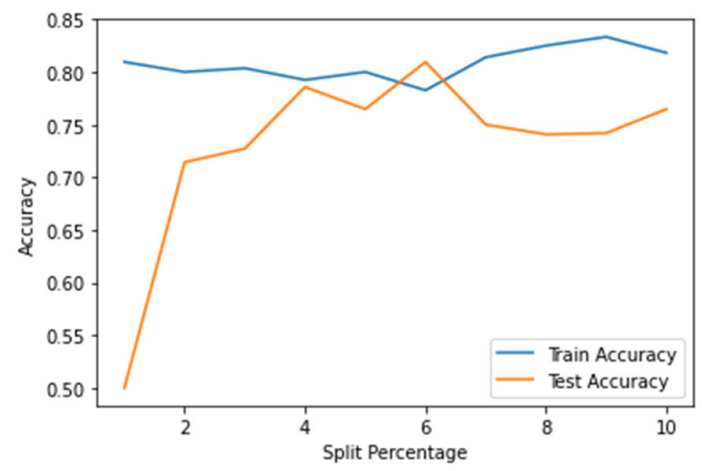

Fig. 17. The accuracy of train and test data for the proposed algorithm

\subsubsection{Test and Train Data Selection}

This research uses the 'train_test_split' command of the sklearn library, the data is divided into two sections where $80 \%$ of the data set will be used for training purposes (24), and $20 \%$ will be considered for training purposes test data (6). Fig. 18 shows the dimensions of the 'Xtrain' and 'Xtest' matrices.

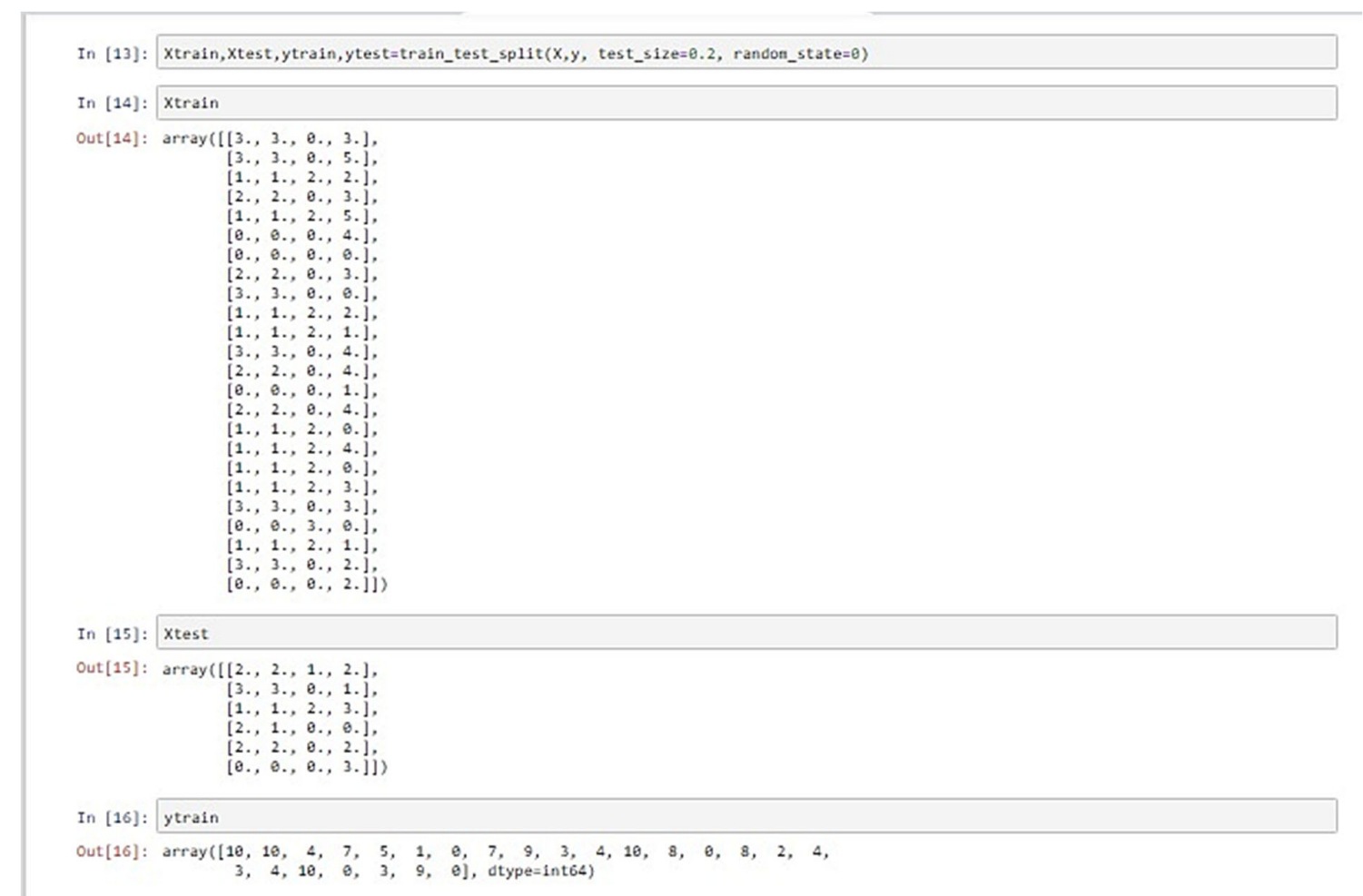

Fig. 18. Clustering dataset into Train and Test Sets

\subsubsection{Learning (Fitting) Process}

In this research, several machine learning algorithms are taken into account. The aim is to find the best machine learning method that can provide a more fitting score and less error for the designed wind turbine. The settings of the classifiers are shown in Table 10. 
Table 10. Settings of the used Supervised Machine-learning Algorithms

\begin{tabular}{lll}
\hline Row & Machine-learning Algorithm & Used Settings \\
\hline $\mathbf{1}$ & Linear Regression & clf1 $=$ linear_model.LinearRegression () \\
$\mathbf{2}$ & Logistic Regression & clf $=$ LogisticRegression(random_state=1, solver='newton-cg', multi_class='multinomial') \\
$\mathbf{3}$ & Random Forest & clf3 $=$ RandomForestClassifier(random_state=3, n_estimators=100) \\
$\mathbf{4}$ & Naive Base $(G a u s s i a n ~ N B)$ & clf4 $=$ GaussianNB () \\
$\mathbf{5}$ & Support Vector Machine & clf5 $=$ SVC $($ gamma='auto' $)$ \\
$\mathbf{6}$ & Multi-Layer Perceptron & clf6 $=$ MLPClassifier(solver='lbfgs', alpha $=1 \mathrm{e}-5$, hidden_layer_sizes $=(8,3)$, random_state=1) \\
$\mathbf{7}$ & Knearest Neighbors & clf7 $=$ KNeighborsClassifier(n_neighbors $=10, \mathrm{p}=2$, metric='minkowski') \\
\hline
\end{tabular}

\subsubsection{Mean Absolute Error and Mean Squared Error}

One crucial step that must be taken after evaluating the performance of the proposed machine-learning model; and before using actual data for predicting the Technological Innovation is to measure the model's accuracy by calculating the errors. For this purpose, Mean Absolute Error and Mean Squared Error is used (Table 11):

$$
\begin{aligned}
& M A E=\left(\frac{1}{n}\right) \sum\left|Y_{\text {test }}-Y_{\text {pred }}\right| \\
& M S E=\left(\frac{1}{n}\right) \sum\left(Y_{\text {test }}-Y_{\text {pred }}\right)^{2}
\end{aligned}
$$

The reason for using both MAE and MSE is that MAE only measures the model's accuracy without considering the direction of the values, and therefore some values may neutralize the effects of other values in the opposite direction. However, it can show the average magnitude of the errors in a set of predictions, and therefore MAE should not be ignored (Table 11).

\subsubsection{Comparing the Performance of Different Supervised Machine-learning Algorithms for the Developed Model}

In this section, the patterns offered by the supervised machine-learning algorithms, including Linear Regression, Logistic Regression, Random Forest, Naive Base (Gaussian NB), Support Vector Machine, Multi-Layer Perceptron, and K-nearest Neighbours will be presented (Table 11).

Table 11. Comparing the Outcomes of Different Supervised Machine-learning Algorithms for the Developed Model

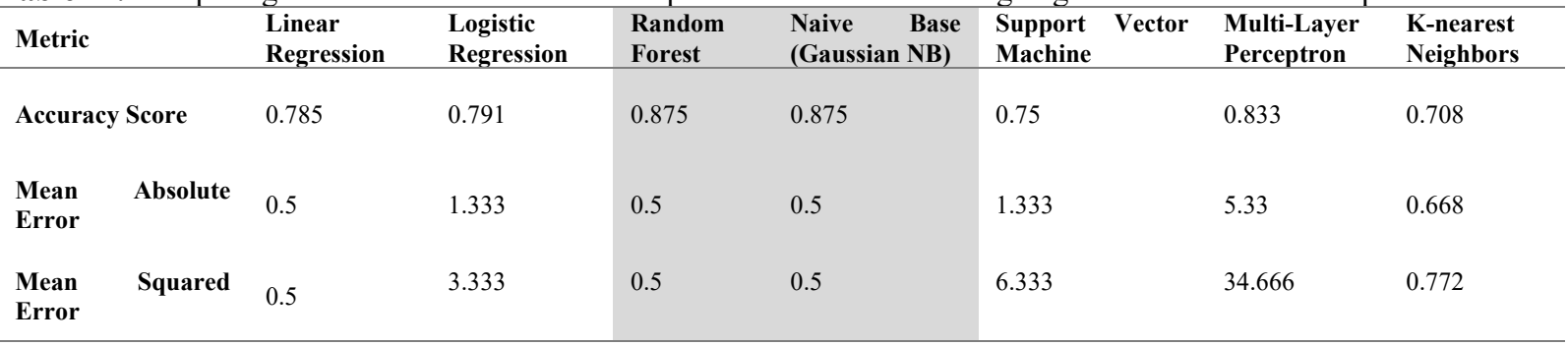

Table 11 presents the results of comparing different supervised machine-learning algorithms indicating Random Forest and Naive Base could provide a base with higher accuracy (0.875) and less Absolute Mean Error and Mean Squared Error at the same time $(0.5$ and 0.5 , respectively). The recognized regions of technological innovation by different machine-learning algorithms are shown in Fig. 19.
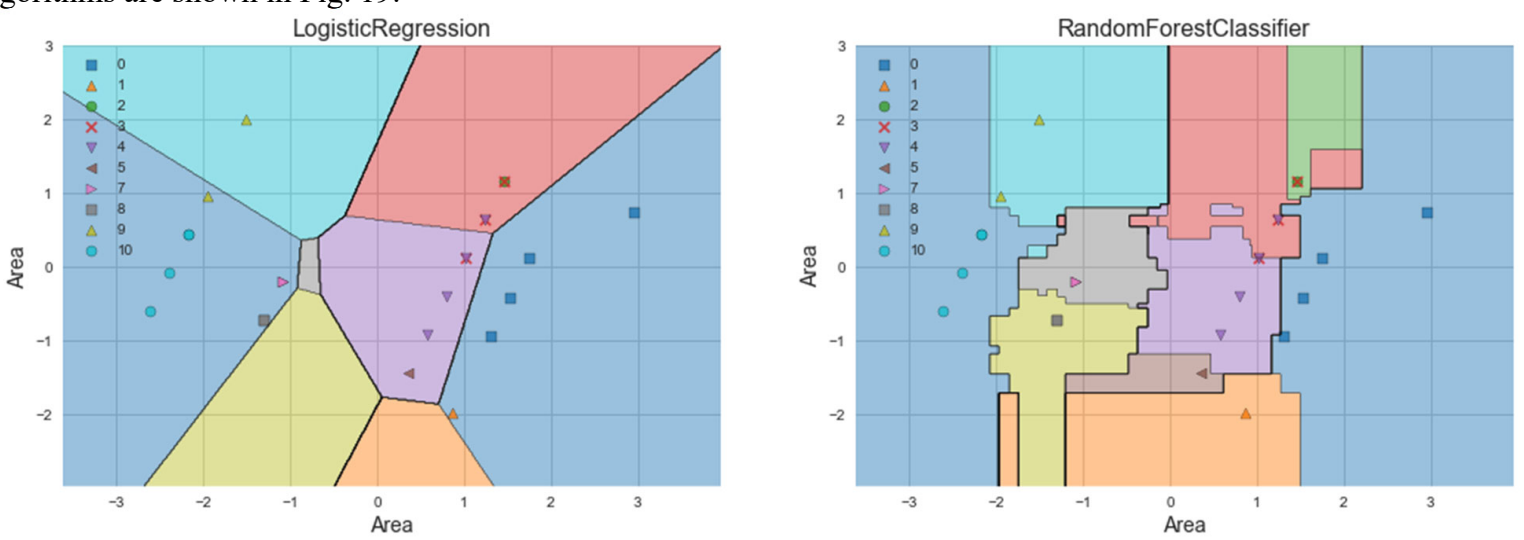

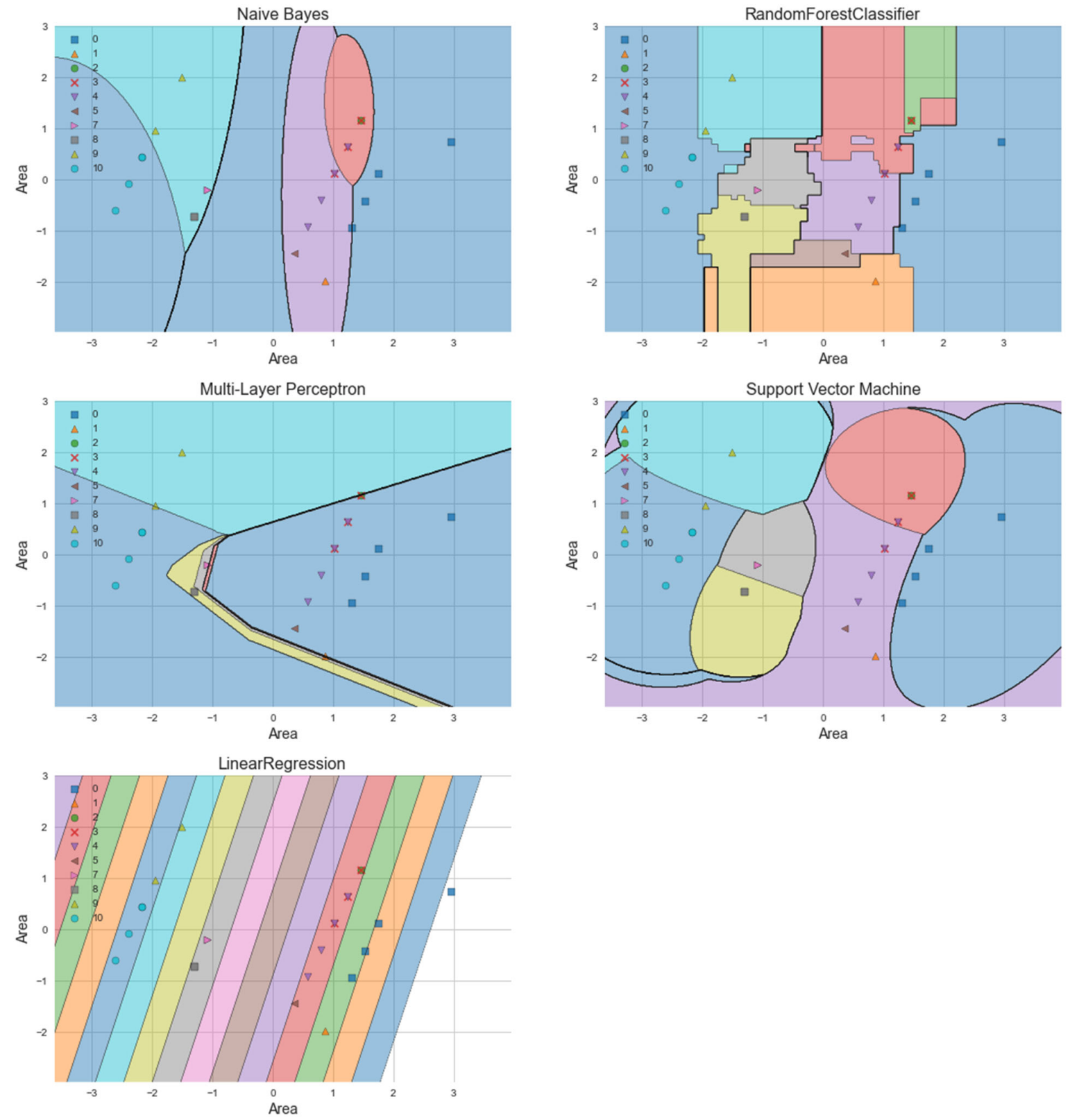

Fig. 19. Comparing Clustered Regions by Different Supervised Machine-learning Algorithms

In Fig. 20, Random Forest Classifier and NB could provide more specific regions. It should be mentioned that the complexity in the plots of Random Forest and BN is due to the complexity of the used data set and not over-fitting. The less accurate method is Linear Regression since the data pattern is very complex and cannot be identified easily by linear lines. Other methods are also provided, but their plots reveal that they are under-fitted. Therefore, using the data used in this model, Random Forest Algorithm seems to draw the best clustering regions.

\subsubsection{Validating the proposed RFC Model}

In order to evaluate the performance of the proposed RFC algorithm for predicting the turbine spin according to the wind speed, several series are given. The outcomes are indicated in Fig. 20.

MAE results indicate that the proposed method could successfully predict and classify most of the validating data (0.871). Using 637 data for the training model, the achievements are noticeable and worthy. Moreover, it can be seen that while the model is confronted with new conditions, which were not defined for it during the training process, it can still successfully determine the class of the technological innovation.

The confusion matrix for the proposed method is shown by Fig. 20: 


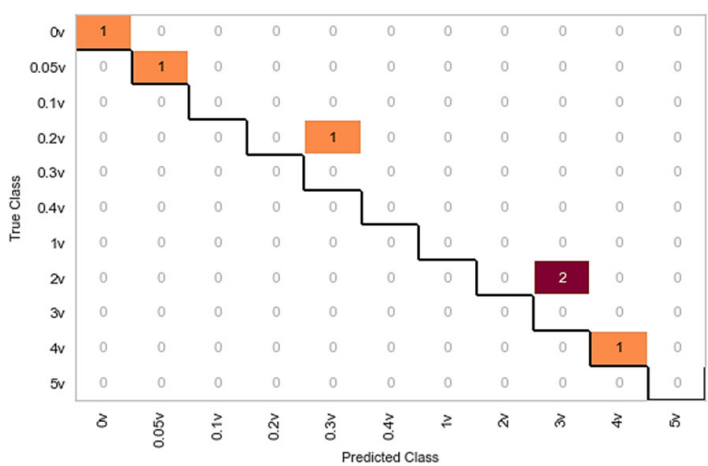

Fig. 20. Confusion matrix for the proposed method

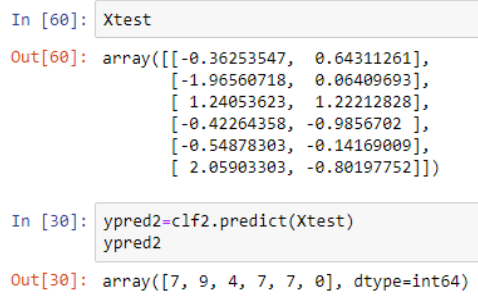

Fig. 21. Dataset for Validating the proposed RFC

Fig. 21 indicated that the proposed method is sensitive to voltage 2 and 3 meaning that in some cases while the real class is $2 \mathrm{v}$, the algorithm might determine it as $3 \mathrm{v}$. However, since in most of the rest cases, the predicted classes are as true classes, the algorithm is reliable enough to be used.

\section{Conclusion}

The efficiency of Vertical Axis Wind Turbines can be improved to give better performance in energy harvesting in terms of its transmission system. Gear ratio plays a vital role in transmission systems, as in motorcars, motorcycles and bicycles. In addition, the centrifugal clutch majorly contributed to the automatic transmission system.

In this research, an automatic transmission system in VAWT is designed. Then, a supervised machine-learning algorithm is proposed for optimizing a designed automatic transmission system for vertical axis wind turbines.

The outcomes indicated that using the machine learning algorithm, the designed automatic transmission system could recognize the wind speed and automatically change its gear ratio by shifting the gears. Consequently, the positive engagement of gears during shifting is achieved which causes the VAWT to spin at the correct gear ratio during the wind speed inconsistency.

The results showed that the application of the automatic transmission system in VAWT has improved energy efficiency significantly (up to $5 \mathrm{~V}$ ) in the Vertical Axis Wind Turbine and reduced energy loss. Furthermore extension by fabricating the system using industrial-level material instead of fabricating using a 3D printer is suggested.

\section{Acknowledgments}

Authors would like to thanks anonymous reviewers and the editor for their efforts during the publication process.

\section{References}

Abbasi, W. A., Wang, Z., Zhou, Y., \& Hassan, S. (2019). Research on measurement of supply chain finance credit risk based on Internet of Things. International Journal of Distributed Sensor Networks, 15(9), 1550147719874002.

Abecassis-Moedas, C. (2006). Integrating design and retail in the clothing value chain: An empirical study of the organisation of design. International Journal of Operations \& Production Management, 26(4), 412-428.

Al-Bahadly, I. (2009). Building a wind turbine for rural home. Energy for sustainable development, 13(3), 159-165.

Ariffin, M., Yee Lee, T., \& Mohamed, S. B. (2014). Design Improvement of Automatic Transmission System for Remote Controlled Car. Paper presented at the Applied Mechanics and Materials.

Asrol, M., Papilo, P., \& Gunawan, F. E. (2021). Support Vector Machine with K-fold Validation to Improve the Industry's Sustainability Performance Classification. Procedia Computer Science, 179, 854-862.

Bhutta, M. M. A., Hayat, N., Farooq, A. U., Ali, Z., Jamil, S. R., \& Hussain, Z. (2012). Vertical axis wind turbine-A review of various configurations and design techniques. Renewable and Sustainable Energy Reviews, 16(4), 1926-1939.

Brito, P. Q., Soares, C., Almeida, S., Monte, A., \& Byvoet, M. (2015). Customer segmentation in a large database of an online customized fashion business. Robotics and Computer-Integrated Manufacturing, 36, 93-100.

Carbonneau, R., Laframboise, K., \& Vahidov, R. (2008). Application of machine learning techniques for supply chain demand forecasting. European Journal of Operational Research, 184(3), 1140-1154.

Castillo, V. E., Mollenkopf, D. A., Bell, J. E., \& Bozdogan, H. (2018). Supply chain integrity: A key to sustainable supply chain management. Journal of Business Logistics, 39(1), 38-56.

Cheng, Y., Peng, J., Gu, X., Zhang, X., Liu, W., Zhou, Z., . . Huang, Z. (2020). An intelligent supplier evaluation model based on data-driven support vector regression in global supply chain. Computers \& Industrial Engineering, 139, 105834.

Cho, J., Jeong, K., Park, M., \& Park, N. (2015). Dynamic response analysis of wind turbine gearbox using simplified local tooth stiffness of internal gear system. Journal of Computational and Nonlinear Dynamics, 10(4), 041001. 
Chong, W., Fazlizan, A., Poh, S., Pan, K., \& Ping, H. (2012). Early development of an innovative building integrated wind, solar and rain water harvester for urban high rise application. Energy and Buildings, 47, 201-207.

Chong, W., Naghavi, M., Poh, S., Mahlia, T., \& Pan, K. (2011). Techno-economic analysis of a wind-solar hybrid renewable energy system with rainwater collection feature for urban high-rise application. Applied Energy, 88(11), 4067-4077.

Chong, W., Pan, K., Poh, S., Fazlizan, A., Oon, C., Badarudin, A., \& Nik-Ghazali, N. (2013). Performance investigation of a power augmented vertical axis wind turbine for urban high-rise application. Renewable Energy, 51, 388-397.

Chong, W., Poh, S., Fazlizan, A., \& Pan, K. (2012). Vertical axis wind turbine with omni-directional-guide-vane for urban high-rise buildings. Journal of Central South University, 19(3), 727-732.

Dabiri, J. O. (2011). Potential order-of-magnitude enhancement of wind farm power density via counter-rotating vertical-axis wind turbine arrays. Journal of renewable and sustainable energy, 3(4), 043104.

Daneshmand-Mehr, M., Najafi, M., \& Sadeghian, R. (2020). Determining the optimal forecasting combination of the four-level

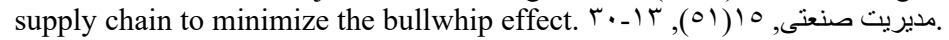

Delgoshaei, A., Ariffin, M. K. A., \& Baharudin, B. (2016). Pre-emptive resource-constrained multimode project scheduling using genetic algorithm: A dynamic forward approach. Journal of Industrial Engineering and Management (JIEM), 9(3), $732-785$.

Delgoshaei, A., \& Gomes, C. (2016). A multi-layer perceptron for scheduling cellular manufacturing systems in the presence of unreliable machines and uncertain cost. Applied Soft Computing, 49, 27-55.

Delgoshaei, A., \& Gomes, C. (2019). A new method for minimizing cell underutilization in the process of dynamic cell forming and scheduling using artificial neural networks. Journal of Advanced Mechanical Design, Systems, and Manufacturing, 13(1), JAMDSM0021-JAMDSM0021.

Eriksson, S., Bernhoff, H., \& Leijon, M. (2008). Evaluation of different turbine concepts for wind power. Renewable and Sustainable Energy Reviews, 12(5), 1419-1434.

Fallahpour, A., Kazemi, N., Molani, M., Nayyeri, S., \& Ehsani, M. (2018). An intelligence-based model for supplier selection integrating data envelopment analysis and support vector machine. Iranian Journal of Management Studies, 11(2), $209-241$.

Fanoodi, B., Malmir, B., \& Jahantigh, F. F. (2019). Reducing demand uncertainty in the platelet supply chain through artificial neural networks and ARIMA models. Computers in biology and medicine, 113, 103415.

Foltin, P., Brunclík, M., Ondryhal, V., \& Vogal, L. (2018). Usability of Performance Indicators of Logistics Infrastructure Availability in Supply Chain Designing. Business Logistics in Modern Management.

Gavalda, J., Massons, J., \& Diaz, F. (1990). Experimental study on a self-adapting Darrieus-Savonius wind machine. Solar \& Wind Technology, 7(4), 457-461.

Gipe, P. (2004). Wind power. Wind Engineering, 28(5), 629-631.

Goettsch, D., Castillo-Villar, K. K., \& Aranguren, M. (2020). Machine-Learning Methods to Select Potential Depot Locations for the Supply Chain of Biomass Co-Firing. Energies, 13(24), 6554.

Golkhoo, F., \& Moselhi, O. (2019). Optimized material management in construction using multi-layer perceptron. Canadian Journal of Civil Engineering, 46(10), 909-923.

Greenblatt, D., Schulman, M., \& Ben-Harav, A. (2012). Vertical axis wind turbine performance enhancement using plasma actuators. Renewable Energy, 37(1), 345-354.

Guo, X., Yuan, Z., \& Tian, B. (2009). Supplier selection based on hierarchical potential support vector machine. Expert Systems with Applications, 36(3), 6978-6985.

Hashim, H., \& Ho, W. S. (2011). Renewable energy policies and initiatives for a sustainable energy future in Malaysia. Renewable and Sustainable Energy Reviews, 15(9), 4780-4787.

Herbert, G. J., Iniyan, S., Sreevalsan, E., \& Rajapandian, S. (2007). A review of wind energy technologies. Renewable and Sustainable Energy Reviews, 11(6), 1117-1145.

Ho, T. K. (1995). Random decision forests. Paper presented at the Proceedings of 3rd international conference on document analysis and recognition.

Hossain, A., Iqbal, A., Rahman, A., Arifin, M., \& Mazian, M. (2007). Design and development of a 1/3 scale vertical axis wind turbine for electrical power generation. Journal of Urban and Environmental Engineering, 1(2), 53-60.

Howell, R., Qin, N., Edwards, J., \& Durrani, N. (2010). Wind tunnel and numerical study of a small vertical axis wind turbine. Renewable Energy, 35(2), 412-422.

Ishak, A., \& Wijaya, T. (2019). Rubber Spare Parts Supplier Selection Model Using Artificial Neural Network: Multi-Layer Perceptron. Paper presented at the 2019 1st International Conference on Engineering and Management in Industrial System (ICOEMIS 2019).

Islam, M., Amin, M. R., Ting, D. S.-K., \& Fartaj, A. (2007). Aerodynamic factors affecting performance of straight-bladed vertical axis wind turbines. Paper presented at the ASME international mechanical engineering congress and exposition.

Islam, M., Ting, D. S.-K., \& Fartaj, A. (2008). Aerodynamic models for Darrieus-type straight-bladed vertical axis wind turbines. Renewable and Sustainable Energy Reviews, 12(4), 1087-1109.

Jiang, M. P., Zheng, S. Y., Wang, H., Zhang, S. Y., Yao, D. S., Xie, C. F., \& Liu, D. L. (2019). Predictive model of aflatoxin contamination risk associated with granary-stored corn with versicolorin A monitoring and logistic regression. Food Additives \& Contaminants: Part A, 36(2), 308-319.

Johari, M. K., Jalil, M., \& Shariff, M. F. M. (2018). Comparison of horizontal axis wind turbine (HAWT) and vertical axis wind turbine (VAWT). International Journal of Engineering and Technology, 7(4.13), 74-80.

Kanellos, F., \& Hatziargyriou, N. (2008). Control of variable speed wind turbines in islanded mode of operation. IEEE Transactions on Energy Conversion, 23(2), 535-543.

Korobenko, A., Hsu, M.-C., Akkerman, I., \& Bazilevs, Y. (2014). Aerodynamic simulation of vertical-axis wind turbines. Journal of Applied Mechanics, 81(2). 
Kozłowski, E., Borucka, A., \& Świderski, A. (2020). Application of the logistic regression for determining transition probability matrix of operating states in the transport systems. Eksploatacja i Niezawodność, 22.

Liu, Y., \& Huang, L. (2020). Supply chain finance credit risk assessment using support vector machine-based ensemble improved with noise elimination. International Journal of Distributed Sensor Networks, 16(1), 1550147720903631.

Mahadevan, S., Poornima, S., Tripathi, K., \& Pushpalatha, M. (2019). A survey on machine learning algorithms for the blood donation supply chain. Paper presented at the Journal of Physics: Conference Series.

Mehrjoo, M., \& Pasek, Z. J. (2014). Impact of product variety on supply chain in fast fashion apparel industry. Procedia CIRP, 17, 296-301.

Mehrolia, S., Alagarsamy, S., \& Solaikutty, V. M. (2021). Customers response to online food delivery services during COVID-19 outbreak using binary logistic regression. International Journal of Consumer Studies, 45(3), 396-408.

Mertens, S., van Kuik, G., \& van Bussel, G. (2003). Performance of an H-Darrieus in the skewed flow on a roof. J. Sol. Energy Eng., $125(4), 433-440$.

Mohamed, M. (2012). Performance investigation of H-rotor Darrieus turbine with new airfoil shapes. Energy, 47(1), 522-530.

Nagurney, A., \& Yu, M. (2012). Sustainable fashion supply chain management under oligopolistic competition and brand differentiation. International Journal of Production Economics, 135(2), 532-540.

Negrutiu, C., Vasiliu, C., \& Enache, C. (2020). Sustainable Entrepreneurship in the Transport and Retail Supply Chain Sector. Journal of Risk and Financial Management, 13(11), 267.

Nivedh, B. (2014). Major failures in the wind turbine components and the importance of periodic inspections. Wind Insid, $2014,5$.

Oh, Y., Busogi, M., Ransikarbum, K., Shin, D., Kwon, D., \& Kim, N. (2019). Real-time quality monitoring and control system using an integrated cost effective support vector machine. Journal of Mechanical Science and Technology, 33(12), 6009-6020.

Park, K.-s., Asim, T., \& Mishra, R. (2012). Computational fluid dynamics based fault simulations of a vertical axis wind turbines. Paper presented at the Journal of Physics: Conference Series.

Polinder, H., Van Bussel, G., \& Dubois, M. (2004). Design of a PM generator for the Turby, a wind turbine for the built environment. Paper presented at the Proceedings of ICEM 2004.

Raut, S. S., \& Mali, D. (2014). Automatic transmission gearbox with centrifugal clutches. International Journal of Research in Engineering and Technology, 3(10), 307-309.

Rezanoori, A., Ariffin, M., Delgoshaei, A., Jalil, N., \& Zulkefli, Z. (2019). A new method to improve passenger vehicle safety using intelligent functions in active suspension system. Engineering Solid Mechanics, 7(4), 313-330.

Shi, Y., Song, X., \& Song, G. (2021). Productivity prediction of a multilateral-well geothermal system based on a long short-term memory and multi-layer perceptron combinational neural network. Applied Energy, 282, 116046.

Silva, N., Ferreira, L. M. D., Silva, C., Magalhães, V., \& Neto, P. (2017). Improving supply chain visibility with artificial neural networks. Procedia Manufacturing, 11, 2083-2090.

Stein, P., Hsu, M.-C., Bazilevs, Y., \& Beucke, K. (2012). Operator-and template-based modeling of solid geometry for Isogeometric Analysis with application to Vertical Axis Wind Turbine simulation. Computer methods in applied mechanics and engineering, 213, 71-83.

Taghiyeh, S., Lengacher, D. C., \& Handfield, R. B. (2020). A Multi-Phase Approach for Product Hierarchy Forecasting in Supply Chain Management: Application to MonarchFx Inc. arXiv preprint arXiv:2006.08931.

Takao, M., Kuma, H., Maeda, T., Kamada, Y., Oki, M., \& Minoda, A. (2009). A straight-bladed vertical axis wind turbine with a directed guide vane row-Effect of guide vane geometry on the performance-. Journal of thermal Science, 18(1), 54-57.

Update, A. M. (2017). Global wind report. Global Wind Energy Council.

Vandenberghe, D., \& Dick, E. (1987). A free vortex simulation method for the straight bladed vertical axis wind turbine. Journal of Wind Engineering and Industrial Aerodynamics, 26(3), 307-324.

Verma, N., \& Pachori, A. (2015). Theoretical Approach for Comparison of Various Types of Wind Generator Systems. International Journal of Recent Resource Electrical and Electronic Engineering. IJRREEE, 2, 29-35.

Villegas, M. A., Pedregal, D. J., \& Trapero, J. R. (2018). A support vector machine for model selection in demand forecasting applications. Computers \& Industrial Engineering, 121, 1-7.

Wan, X.-1., Zhang, Z., Rong, X.-X., \& Meng, Q.-c. (2016). Exploring an interactive value-adding data-driven model of consumer electronics supply chain based on least squares support vector machine. Scientific Programming, 2016.

$\mathrm{Wu}$, Q. (2010). Product demand forecasts using wavelet kernel support vector machine and particle swarm optimization in manufacture system. Journal of computational and applied mathematics, 233(10), 2481-2491.

Yeh, T.-H., \& Wang, L. (2008). A study on generator capacity for wind turbines under various tower heights and rated wind speeds using Weibull distribution. IEEE Transactions on Energy Conversion, 23(2), 592-602.

Yue, L., Yafeng, Y., Junjun, G., \& Chongli, T. (2007). Demand forecasting by using support vector machine. Paper presented at the Third International Conference on Natural Computation (ICNC 2007).

Zhang, Y. (2019). Application of improved BP neural network based on e-commerce supply chain network data in the forecast of aquatic product export volume. Cognitive Systems Research, 57, 228-235.

Zhou, E., Zhang, J., Gou, Q., \& Liang, L. (2015). A two period pricing model for new fashion style launching strategy. International Journal of Production Economics, 160, 144-156.

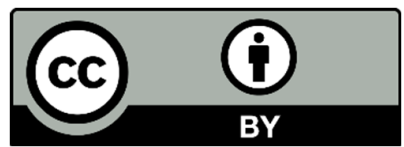

(C) 2022 by the authors; licensee Growing Science, Canada. This is an open access article distributed under the terms and conditions of the Creative Commons Attribution (CC-BY) license (http://creativecommons.org/licenses/by/4.0/). 